\title{
A family of term-structure models for long-term risk management and derivative pricing
}

\author{
Andrew J.G. Cairns \\ Department of Actuarial Mathematics and Statistics, \\ Heriot-Watt University, \\ Edinburgh, EH14 4AS, United Kingdom \\ E-mail: A.Cairns@ma.hw.ac.uk \\ Internet: www.ma.hw.ac.uk/ andrewc/ \\ First version: February 26, 1999 \\ This version: March 21, 2003
}

\begin{abstract}
In this paper we propose a new family of term-structure models based upon the Flesaker \& Hughston (1996) positive-interest framework. We demonstrate that the models are ideally suited for use in long-term risk management as well as for shortterm derivative-pricing problems. In particular, the models can be parametrised in a way which gives sustained periods of both high and low interest rates, similar to the cycle lengths we have observed over the course of the 20th century in the UK and US.
\end{abstract}

Keywords: term-structure model; multifactor; positive interest; Ornstein-Uhlenbeck; time-homogeneous; Japan; dynamic financial analysis. 


\section{Introduction}

In this paper we propose a new family of models for the term-structure of interest rates. A primary motivation for the development of these models was the perceived need for new models which combine the two characteristics:

- reasonable, arbitrage-free dynamics in the short term;

- realistic dynamics in the long term.

Recent years have seen considerable research and development of models dealing with the first of these characteristics (see, for example, the models or frameworks proposed by Vasicek, 1977, Cox, Ingersoll \& Ross, 1985, Black \& Karasinski, 1991, Heath, Jarrow \& Morton, 1992, Brace, Gatarek \& Musiela, 1997 and Duffie \& Kan, 1996, or the books by James \& Webber, 2000, and Cairns, 2003). Development of these models has been driven primarily by the need for models to price and hedge relatively short-term interest-rate derivatives.

On the other hand a number of models have been developed, mainly in an insurance context, which address the second characteristic (for example, Wilkie, 1995, Yakoubov, Teeger \& Duval, 1999). These models are, strictly, arbitrage free but they are typically framed in discrete time and were not designed for short-term risk management or derivative pricing. These models were developed because of the clear need within life insurance and pensions to assess long-term asset and liability risks.

Until very recently very little research has been conducted into the development of models which satisfy both of these characteristics. These recent developments have now become very important as interest rates in the early 2000's reached historical lows. In particular, this has resulted in substantial increases in the value of interestrate guarantees issued by insurers on a variety of contracts (see, for example, Waters, Wilkie \& Yang, 2003). Besides the insurers' need for good internal models, banks have also developed a need for good long-term interest-rate models as demand from insurers for certain long-maturity derivatives has increased. Additionally, changes in accounting practices have led to a requirement for interest-rate models which can deal with pricing (the so-called "fair value" of liabilities) as well as long-term risk management.

\subsection{Data}

Before we describe the main contents of this paper we will take a brief look at some historical data to provide us with some pointers towards desirable characteristics of an interest-rate model. 
In Figures 1.1 and 1.3 we have plotted the development over time of short and longterm interest rates in the UK and the USA. In Figures 1.2 and 1.4 short-term rates are plotted against long-term rates. We can make the following observations:

- Short and long-term interest rates are highly, but imperfectly correlated in the medium and long term. In the shorter term the rates are still correlated, but to a smaller extent.

- Since the short-term and long-term interest rates are not perfectly correlated (comonotonic), the data are clearly inconsistent with the use of a one-factor, time-homogeneous model (as considered by Chan et al., 1992).

- Short-term interest rates are more volatile.

- Short and long-term interest rates are subject to sustained periods of both high (for example, 1970-1990 in the UK) and low interest rates (UK: 1900-1960).

We can also remark that the existence of such long cycles in interest rates makes it essentially impossible to test for stationarity in the various series.

- Both short and long-term interest rates have varied over a considerable range (UK: $2 \%$ to $17 \%$; USA: $1 \%$ to $17 \%$ ).

In general, although not always, continuous-time, arbitrage-free models include a degree of mean reversion which is too strong to allow for long cycles without compromising the short-term dynamics. A family of models which provides an exception to this is the multifactor CIR class (see, for example, Longstaff \& Schwartz, 1992, or Duffie, 1996, Chapter 7). However, these models have the problem that all interest rates other than the risk-free rate, $r(t)$, have strictly positive lower bounds, which compromises the pricing of derivatives which come into the money when interest rates are low. Additionallt this class of models cannot reproduce the sort of yield curve experienced in Japan in 2002 where interest rates out to several years' maturity are very low (similar to curve F in Figure 4.1).

Based on these observations we can draw up the usual list of desirable characteristics for a term-structure model, supplemented by others which fit in with the aim of using a model for long-term risk management.

1. The model should be arbitrage free to allow its use in derivative pricing and short-term dynamic hedging.

2. All interest rates should be positive and all rates should be able to take values arbitrarily close to zero (albeit with very small probability).

3. The model should be framed in continuous time. This allows implementation in discrete time with any length of time step, $\Delta t$, without the need to construct a new model each time we change $\Delta t$. 


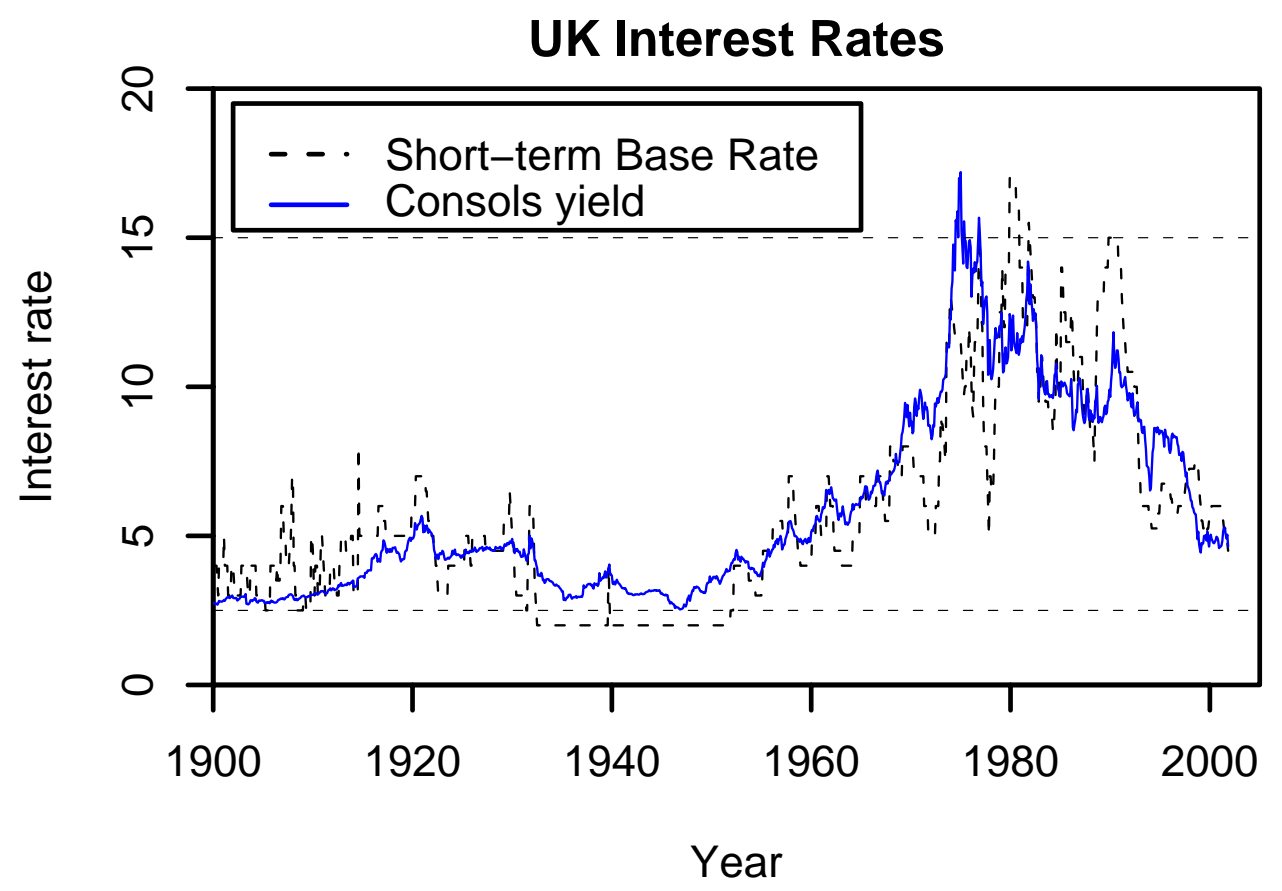

Figure 1.1: UK interest rates 1900-2002. Bank of England Base Rate (short-term) and UK Consol's yields (perpetual bonds) plotted against time.

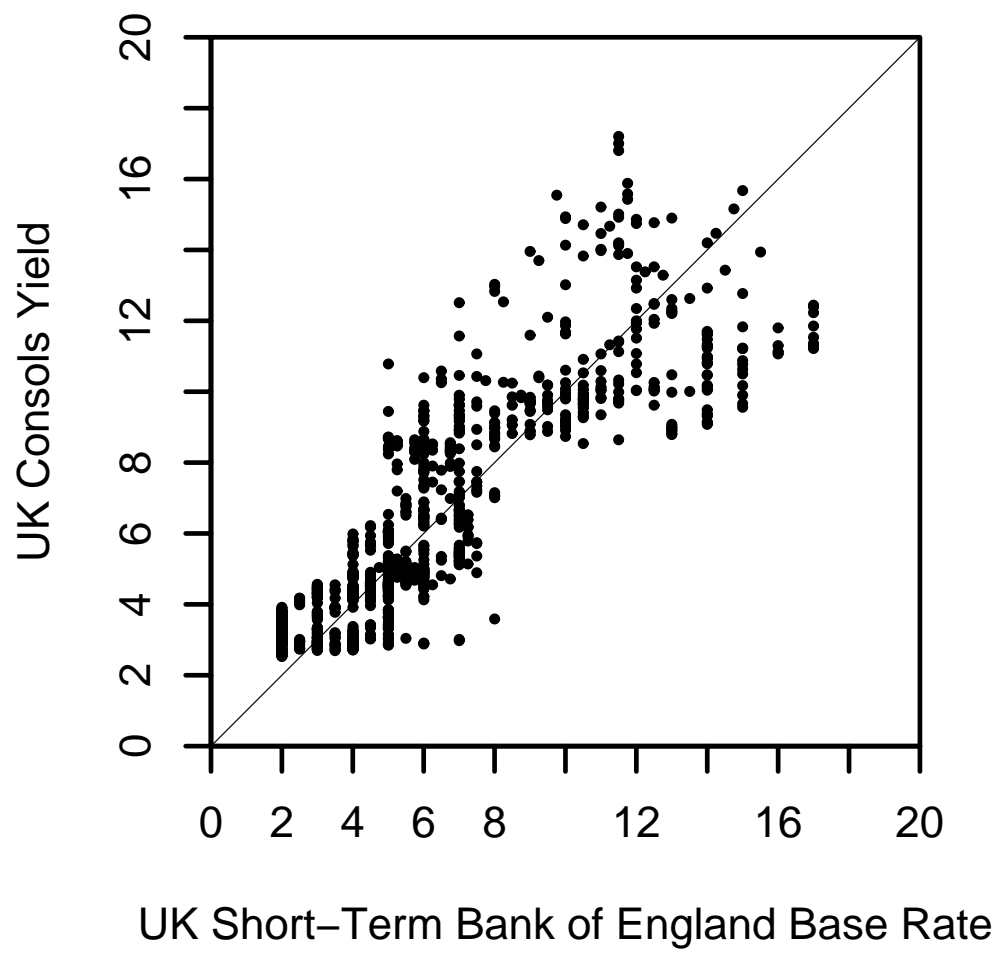

Figure 1.2: UK interest rates 1900-2002. Short-term versus long-term interest rates. 


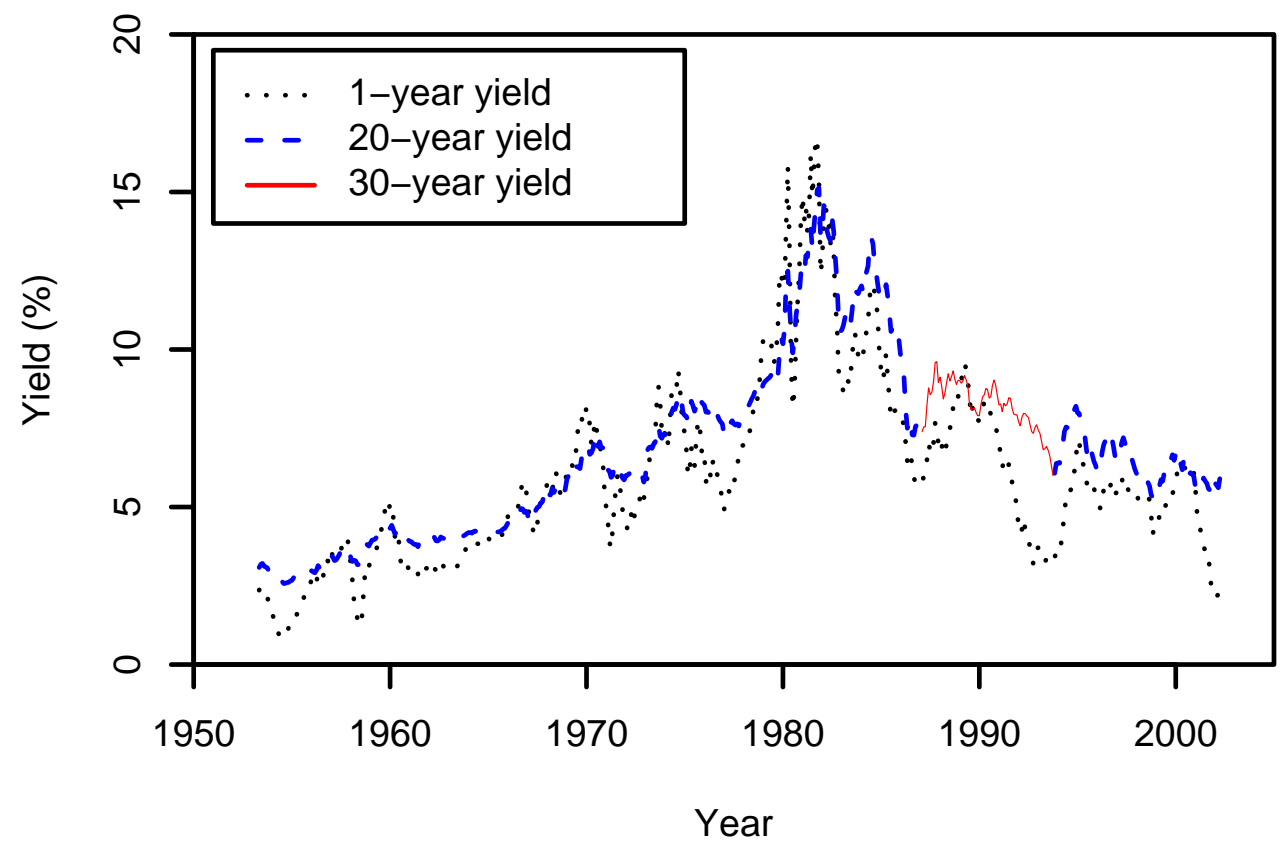

Figure 1.3: US interest rates 1954-2002. 1-year interest rates and long-bond yields. Long bond yields are mostly 20-year par yields with 30-year yields to fill in a gap in the 1980's.

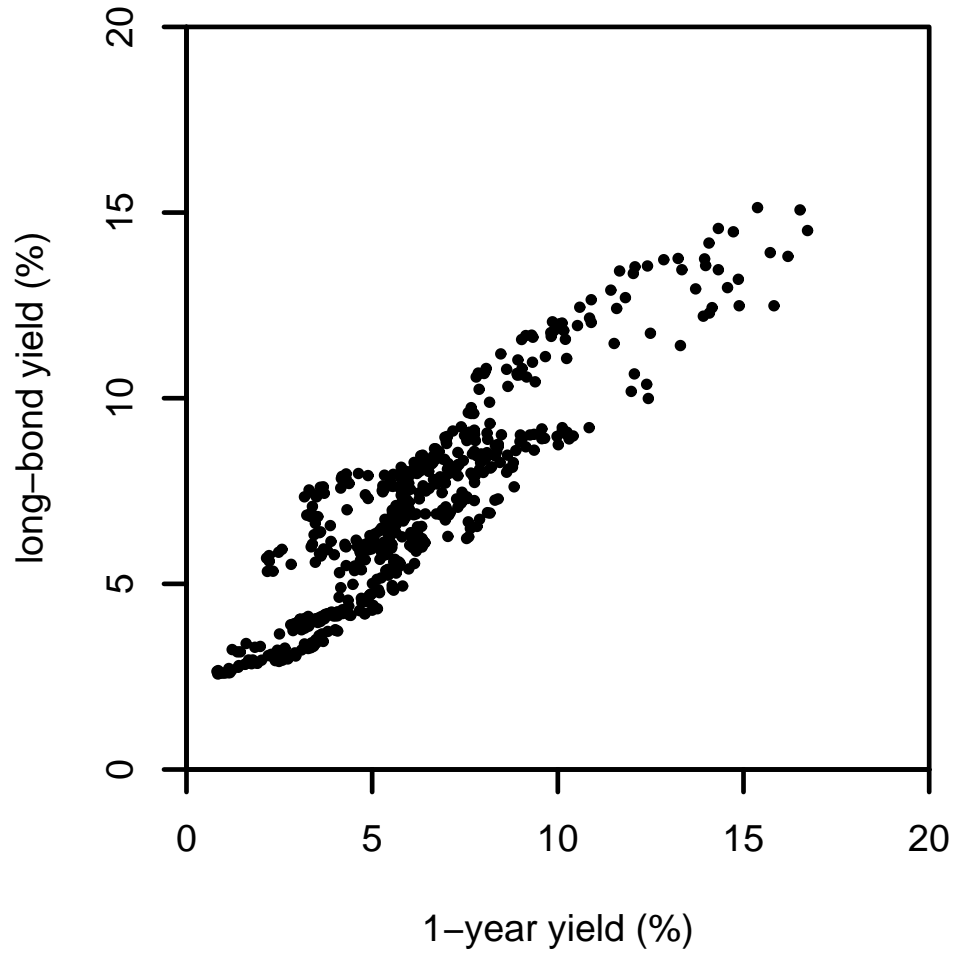

Figure 1.4: US interest rates 1954-2002. Short-term versus long-term interest rates. 
4. Short-term interest-rate dynamics should be consistent with what we observe in historical data.

5. The model should permit straightforward analytical or numerical calculation of bond and derivative prices.

6. Interest rates should be mean reverting. This requirement reflects the perception that whenever interest rates reach extreme levels the government will intervene in order to bring rates back to more reasonable levels rather than let rates continue unchecked to greater extremes.

7. The model should be flexible enough (a) to give rise to a range of different yield curve shapes and (b) to be able to deal with a variety of derivative contracts.

8. The model should give rise to sustained periods of both high and low interest rates of all maturities, and in a way which is consistent with what we have observed in the past.

9. Interest rates with different terms to maturity should, with reasonable probability, be able to attain both high and low values consistent with what we have observed in historical data (for example, the $2 \%$ to $17 \%$ observed in UK data).

10. Going beyond consistency with historical data, the model should fit historical data adequately in the statistical sense.

The last of these charateristics is one which we leave for future work. However, we can make some observations relating to this point. If the model is to be used for long-term risk-management purposes then it is appropriate to fit the model using historical data. This may be done with backup from traded-derivative prices if required. For example, historical interest-rate data may result in very wide confidence intervals for some parameters. These confidence intervals can sometimes be reduced by incorporating information from the derivatives market. However, as with all modelling exercises, we are subject to both model risk and parameter uncertainty. In general parameter uncertainty tends to be the more important source of error for long-term risk management. This means that probability statements derived from the use of a single model and parameter set should be treated with caution. However, what is important in any modelling exercise is that we have a model which gives rise to a wide and realistic range of future scenarios. (For example, it is important that long cycles can occur and that interest rates can exceed $15 \%$ from time to time with reasonable probability rather than not at all. It is much less important to be able to say that a particular event will happen with probability exactly 0.01. It could equally be 0.005 or 0.04 .) The aim is then to identify which types of scenario pose the greatest risk to a financial institution and which do not, and then act accordingly to reduce these risks. 


\subsection{Outline of the paper}

We will now move on to the development of a family of models which address all these desirable charateristics.

In Section 2 we review, briefly, the general, positive-interest framework proposed by Flesaker \& Hughston (1996) (FH), Rogers (1997) and Rutkowski (1997). We then use the $\mathrm{FH}$ approach to propose a general family of multifactor models for the term structure with a multivariate Ornstein-Uhlenbeck process, $X(t)$, as its driver. In particular, we will see that zero-coupon bond prices can be expressed in the time-homogeneous form

$$
P(t, T)=\frac{\int_{T-t}^{\infty} H(u, X(t)) d u}{\int_{0}^{\infty} H(u, X(t)) d u}
$$

for some function $H(u, x)$.

In Section 3 we prove certain theoretical properties of the one-factor version of the model. In particular, we prove its asymptotic similarity to the Black \& Karasinski (1991) model when the risk-free rate $r(t)$ gets very small and to the Vasicek model when $r(t)$ gets very large.

In Section 4 we provide an extensive analysis of a two-factor version of the model. Here we make a variety of qualitative observations based on accurate numerical calculations of bond prices and interest rates. In particular, we aim to demonstrate that the new family of models satisfies all of the desirable charateristics 1 to 9 if we choose our parameters in an appropriate way.

In the final Section 5 we propose a extension of the family of models analysed in this paper similar to Duffie \& Kan's (1996) exposition on affine term-structure models.

\section{A model for fixed-interest bond prices}

\section{$2.1 \quad$ Background}

In this section we propose a family of multifactor models for the fixed-interest term structure which makes use of the framework developed by Flesaker \& Hughston (1996) (FH), and later extended by Rutkowski (1997) and Rogers (1997).

We define $P(t, T)$ to be the price at time $t$ of a zero-coupon bond which matures at time $T$. Under the $\mathrm{FH}$ framework the dynamics of these prices are linked to the progress of a family of martingales, $M(t, s)$, under some reference or pricing measure $\hat{P}$.

Let $M(t, s)$ for $0 \leq t \leq s<\infty$ be a family of strictly-positive diffusion processes over the index $s$ which are martingales with respect to $t$ under some probability measure 
$\hat{P}$ : that is, given $s$, for $t<u<s, E_{\hat{P}}\left[M(u, s) \mid \mathcal{F}_{t}\right]=M(t, s)$. Furthermore, we define $M(0, s)=1$ for all $s$ and we will assume in this paper that for each $s, M(t, s)$ is a diffusion process adapted to a finite ( $\operatorname{say} n$ ) dimensional Brownian motion, $\hat{Z}(t)$ (under $\hat{P}$ ).

FH proposed that zero-coupon bond prices are defined by the equation

$$
P(t, T)=\frac{\int_{T}^{\infty} M(t, s) \phi(s) d s}{\int_{t}^{\infty} M(t, s) \phi(s) d s}
$$

for some deterministic function $\phi(s)$.

A more general form of this framework was proposed by Rutkowski (1997) and Rogers (1997). Rutkowski (1997) defines

$$
P(t, T)=\frac{E_{\hat{P}}\left[A(T) \mid \mathcal{F}_{t}\right]}{A(t)}
$$

where $A(t)$ is a strictly-positive supermartingale under the measure $\hat{P}$. For this reason we will often refer to $\hat{P}$ as the pricing measure. The $\mathrm{FH}$ form which we will use in this paper is clearly a special case of this framework, where $A(t)=$ $\int_{t}^{\infty} M(t, s) \phi(s) d s$. Rutkowski (1997) and Rogers (1997) demonstrate that models of this general type are arbitrage free. These developments extended earlier work by Constantinides (1992) where, amongst other differences, the pricing measure $\hat{P}$ was identified with the real-world measure $P$.

Where the objective is to price a derivative contract which pays $V(T)$ at time $T$ the price (using the Rutkowski, 1997, formulation) at time $t$ is

$$
V(t)=\frac{E_{\hat{P}}\left[A(T) V(T) \mid \mathcal{F}_{t}\right]}{A(t)} .
$$

In this paper we will make use of the $\mathrm{FH}$ formulation (equation 2.1) in our development of the new family of models.

Since $M(0, s)=1$ for all $s$ we may infer that, if we choose to calibrate initial theoretical to observed prices, we require

$$
\phi(s)=\frac{\partial}{\partial s} P(0, s)
$$

up to a constant, non-zero scaling factor.

Instantaneous forward rates are then

$$
\begin{aligned}
& f(t, T)=-\frac{\partial}{\partial T} \log P(t, T)=\frac{M(t, T) \phi(T)}{\int_{T}^{\infty} M(t, s) \phi(s) d s} \\
& \Rightarrow r(t)=f(t, t)=\frac{M(t, t) \phi(t)}{\int_{t}^{\infty} M(t, s) \phi(s) d s} .
\end{aligned}
$$


Although we can write down an expression for the short rate, $r(t)$, in this way it is not possible, in general, to express the dynamics of $r(t)$ in any simple fashion (for example, like we can with the Vasicek, 1977, model).

We can also write down expressions for bond volatilities which enables us to link the model into the framework of Heath, Jarrow \& Morton (1992). Since $M(t, T)$ is a martingale under $\hat{P}$ for each $T$, we can write $d M(t, T)=M(t, T) \sigma(t, T)^{\prime} d \hat{Z}(t)$ where $\hat{Z}(t)$ is a standard $n$-dimensional Brownian motion under $\hat{P}$ and $\sigma(t, T)$ (for fixed $T$ ) is a a previsible $n \times 1$ vector process. We now define the $n \times 1$ vector

$$
V(t, T)=\frac{\int_{T}^{\infty} M(t, s) \phi(s) \sigma(t, s) d s}{\int_{T}^{\infty} M(t, s) \phi(s) d s} .
$$

The dynamics of the zero-coupon bond prices can then be expressed in the form

$$
\begin{aligned}
\frac{d P(t, T)}{P(t, T)} & =r(t) d t+S_{P}(t, T)^{\prime}(d \hat{Z}(t)-V(t, t) d t) \\
\text { where } S_{P}(t, T) & =V(t, T)-V(t, t)
\end{aligned}
$$

(see, for example, Cairns, 2003).

It follows that the vector $S_{P}(t, T)$ is the price volatility function with each of its $n$ components defining the volatility of the price of a particular bond with respect to each of the $n$ sources of uncertainty.

Since we have expressed the price dynamics in the way given above we can immediately see that if

$$
\tilde{Z}(t)=\hat{Z}(t)-\int_{0}^{t} V(s, s) d s
$$

then $d P(t, T)=P(t, T)\left(r(t) d t+S_{P}(t, T)^{\prime} d \tilde{Z}(t)\right)$.

Suppose that $\sigma(t, T)$ has been defined in such a way that the Novikov condition

$$
E_{\hat{P}}\left[\exp \left(\frac{1}{2} \int_{0}^{t} \sum_{i=1}^{n} V_{i}(s, s)^{2} d s\right)\right]<\infty
$$

is satisfied (see, for example, Karatzas \& Shreve, 1998). Then, by the CameronMartin-Girsanov (CMG) Theorem, there exists a measure $Q$ equivalent to $\hat{P}$ under which $\tilde{Z}(t)$ is an $n$-dimensional Brownian motion. Given the form of $d P(t, T)$ we can see that $Q$ is the usual risk-neutral measure. In particular, if each $\sigma_{i}(t, T)$ for all $t, T>t$, is bounded, then we can see from equation (2.6) that $V_{i}(s, s)$ must be bounded, so the Novikov condition is satisfied.

We can also consider the dynamics of the forward-rate curve $f(t, T)$ under $\hat{P}$ and $Q$. Since $f(t, T)=M(t, T) \phi(T) / \int_{T}^{\infty} M(t, s) \phi(s) d s$ a straightforward application of 
Ito's Lemma and the Product Rule gives us

$$
\begin{aligned}
d f(t, T) & =f(t, T)(\sigma(t, T)-V(t, T))^{\prime}\{d \hat{Z}(t)-V(t, T) d t\} \\
& =f(t, T)(\sigma(t, T)-V(t, T))^{\prime}\left\{d \tilde{Z}(t)-S_{P}(t, T) d t\right\} .
\end{aligned}
$$

\section{$2.2 \quad$ A specific multifactor model}

We modify now the earlier representation of $M(t, T)$

$$
\begin{aligned}
M(0, T) & =1 \text { for all } T \\
d M(t, T) & =M(t, T) \sigma(t, T)^{\prime} d \hat{Y}(t)=M(t, T) \sigma(t, T)^{\prime} C d \hat{Z}(t) \\
& =M(t, T) \sum_{i=1}^{n} \sigma_{i}(t, T) d \hat{Y}_{i}(t) \\
\text { where } d \hat{Y}(t) & =C d \hat{Z}(t) \\
\hat{Y}(0) & =0
\end{aligned}
$$

and $\hat{Z}_{1}(t), \ldots, \hat{Z}_{n}(t)$ are $n$ independent Brownian motions under $\hat{P}$. In this expression we choose the matrix $C$ such that $C C^{\prime}=\left(\rho_{i j}\right)_{i, j=1}^{n}$ is an instantaneous correlation matrix. It follows then that each of the $\hat{Y}_{i}(t)$ is a Brownian motion under $\hat{P}$ with $d\left\langle\hat{Y}_{i}(t), \hat{Y}_{j}(t)\right\rangle=\rho_{i j} d t$.

Suppose now that $\sigma_{i}(t, T)=\sigma_{i} \exp \left[-\alpha_{i}(T-t)\right]$. Then

$$
\begin{aligned}
d \log M(t, T) & =\sigma(t, T)^{\prime} d \hat{Y}(t)-\frac{1}{2} \sum_{i, j=1}^{n} \sigma_{i}(t, T) \sigma_{j}(t, T) d\left\langle\hat{Y}_{i}(t), \hat{Y}_{j}(t)\right\rangle \\
\Rightarrow \log M(t, T) & =\sum_{i=1}^{n} \sigma_{i} \int_{0}^{t} e^{-\alpha_{i}(T-s)} d \hat{Y}_{i}(s)-\frac{1}{2} \sum_{i, j=1}^{n} \rho_{i j} \sigma_{i} \sigma_{j} \int_{0}^{t} e^{-\left(\alpha_{i}+\alpha_{j}\right)(T-s)} d s \\
& =\sum_{i=1}^{n} \sigma_{i} e^{-\alpha_{i}(T-t)} \hat{X}_{i}(t)-\frac{1}{2} \sum_{i, j=1}^{n} \frac{\rho_{i j} \sigma_{i} \sigma_{j}}{\alpha_{i}+\alpha_{j}} e^{-\left(\alpha_{i}+\alpha_{j}\right)(T-t)}\left(1-e^{-\left(\alpha_{i}+\alpha_{j}\right) t}\right)
\end{aligned}
$$

where $\hat{X}_{i}(t)=\int_{0}^{t} \exp \left[-\alpha_{i}(t-s)\right] d \hat{Y}_{i}(s)$ is an Ornstein-Uhlenbeck process with $\hat{X}_{i}(0)=0$ and $d \hat{X}_{i}(t)=-\alpha_{i} \hat{X}_{i}(t) d t+d \hat{Y}_{i}(t)$. Since the $\hat{Y}_{i}(t)$ are correlated so are the $\hat{X}_{i}(t)$.

In contrast to the FH approach for $\phi(s)$ (equation (2.3)) we define

$$
\phi(s)=\phi \exp \left[-\beta s+\sum_{i=1}^{n} \sigma_{i} \hat{x}_{i} e^{-\alpha_{i} s}-\frac{1}{2} \sum_{i, j=1}^{n} \frac{\rho_{i j} \sigma_{i} \sigma_{j}}{\alpha_{i}+\alpha_{j}} e^{-\left(\alpha_{i}+\alpha_{j}\right) s}\right]
$$

for some parameters $\phi, \beta, \hat{x}_{1}, \ldots, \hat{x}_{n}$. (Of these parameters we will see that, provided $\phi \neq 0$, the actual value of $\phi$ is irrelevant. On the other hand $\beta$ has an unambiguous 
interpretation (as we will see in Section 2.3) since it is equal to the constant longterm forward rate.) Then for $t<s$

$$
\phi(s) M(t, s)=\phi \exp \left[-\beta s+\sum_{i=1}^{n} \sigma_{i} e^{-\alpha_{i}(s-t)} X_{i}(t)-\frac{1}{2} \sum_{i, j=1}^{n} \frac{\rho_{i j} \sigma_{i} \sigma_{j}}{\alpha_{i}+\alpha_{j}} e^{-\left(\alpha_{i}+\alpha_{j}\right)(s-t)}\right]
$$

where $X_{i}(t)=\hat{x}_{i} \exp \left(-\alpha_{i} t\right)+\hat{X}_{i}(t)$. From this we can see that the complex choice for $\phi(s)$ ensures that several terms in $\phi(s)$ and $M(t, s)$ cancel. $X_{i}(t)$ is an OrnsteinUhlenbeck process under $\hat{P}$ with $X_{i}(0)=\hat{x}_{i}$ and $d X_{i}(t)=-\alpha_{i} X_{i}(t) d t+d \hat{Y}_{i}(t)$.

It follows that

$$
\begin{aligned}
A(t, T) & =\int_{T}^{\infty} \phi(s) M(t, s) d s \\
& =\phi e^{-\beta t} \int_{T-t}^{\infty} H(u, X(t)) d u \\
\text { where } H(u, x) & =\exp \left[-\beta u+\sum_{i=1}^{n} \sigma_{i} x_{i} e^{-\alpha_{i} u}-\frac{1}{2} \sum_{i, j=1}^{n} \frac{\rho_{i j} \sigma_{i} \sigma_{j}}{\alpha_{i}+\alpha_{j}} e^{-\left(\alpha_{i}+\alpha_{j}\right) u}\right] .
\end{aligned}
$$

We then have

$$
P(t, T)=\frac{A(t, T)}{A(t, t)}=\frac{\int_{T-t}^{\infty} H(u, X(t)) d u}{\int_{0}^{\infty} H(u, X(t)) d u}
$$

Other specific models using Ornstein-Uhlenbeck processes as drivers within this positive-interest framework have been proposed by Rogers (1997).

\subsection{Forward rates and irredeemable bond yields}

We will now look at forward rates for this model and demonstrate that $f(t, T) \rightarrow \beta$ as $T \rightarrow \infty$.

Applying the general formula in equation (2.4) we see that the forward-rate curve 
is

$$
\begin{aligned}
f(t, T)= & \frac{H(T-t, X(t))}{\int_{T-t}^{\infty} H(u, X(t)) d u} \\
= & \left\{\int _ { T - t } ^ { \infty } \operatorname { e x p } \left(-\beta\{u-(T-t)\}+\sum_{i=1}^{n} \sigma_{i} X_{i}(t)\left[e^{-\alpha_{i} u}-e^{-\alpha_{i}(T-t)}\right]\right.\right. \\
\left.\left.-\frac{1}{2} \sum_{i, j=1}^{n} \frac{\rho_{i j} \sigma_{i} \sigma_{j}}{\alpha_{i}+\alpha_{j}}\left[e^{-\left(\alpha_{i}+\alpha_{j}\right) u}-e^{-\left(\alpha_{i}+\alpha_{j}\right)(T-t)}\right]\right) d u\right\}^{-1} & \\
= & \left\{\int _ { 0 } ^ { \infty } \operatorname { e x p } \left(-\beta v+\sum_{i=1}^{n}\left\{\sigma_{i} X_{i}(t) e^{-\alpha_{i}(T-t)}\left(e^{-\alpha_{i} v}-1\right)\right.\right.\right. \\
\Rightarrow r(t)= & \left\{\int_{0}^{\infty} \exp \left(-\beta v+\sum_{i, j=1}^{n} \frac{\rho_{i j} \sigma_{i} \sigma_{j}}{\alpha_{i}+\alpha_{j}} e^{-\left(\alpha_{i}+\alpha_{j}\right)(T-t)}\left[e^{-\left(\alpha_{i}+\alpha_{j}\right) v}-1\right]\right) d v\right\}^{-1} \\
\left.\left.-\frac{1}{2} \sum_{i, j=1}^{n} \frac{\rho_{i j} \sigma_{i} \sigma_{j}}{\alpha_{i}+\alpha_{j}}\left[e^{-\left(\alpha_{i}+\alpha_{j}\right) v}-1\right]\right) d v\right\}^{-1} &
\end{aligned}
$$

If we look more closely at the formula for $f(t, T)$ we can see that as $T$ tends to infinity, the terms in the summations tend to zero. This implies that as $T$ tends to infinity $f(t, T)$ tends to $\beta$ : that is, $\beta$ is the constant long-term forward rate. (This is consistent with a result of Dybvig, Ingersoll \& Ross, 1994. They established that, under the assumption of no arbitrage, a model for the term-structure of interest rates must have a non-decreasing long-term spot rate.)

The par yield on irredeemable bonds (assuming continuous payment of coupons) is

$$
\rho(t)=\left[\int_{0}^{\infty} P(t, t+s) d s\right]^{-1}=\frac{\int_{0}^{\infty} H(u, X(t)) d u}{\int_{0}^{\infty} u H(u, X(t)) d u} .
$$

We consider the process for $\rho(t)$ in a later section when we look at the qualitative properties of some specific models. 


\subsection{Equivalence of $\hat{P}$ and $Q$}

Recall that $d \tilde{Z}_{j}(t)=d \hat{Z}_{j}(t)-V_{j}(t, t) d t$ where the $\tilde{Z}_{j}(t)$ and $\hat{Z}_{j}(t)$ are Brownian Motions under the risk-neutral measure $Q$ and the pricing measure $\hat{P}$ respectively. Here

$$
\begin{aligned}
V_{j}(t, t) & =\frac{\int_{0}^{\infty} H(u, X(t)) \bar{\sigma}_{j}(u) d u}{\int_{0}^{\infty} H(u, X(t)) d u} \\
\text { where } \bar{\sigma}_{j}(u) & =\sum_{i=1}^{n} \sigma_{i} e^{-\alpha_{i} u} c_{i j} .
\end{aligned}
$$

Since $H(u, x)>0$ for all $u>0,-\infty<x<\infty$, we have $V_{j}(t, t)<\sum_{i=1}^{n}\left|\sigma_{i} c_{i j}\right|<\infty$ for all $t$. This upper bound implies that $E_{\hat{P}}\left[\exp \left(\frac{1}{2} \int_{0}^{t} \sum_{j=1}^{n} V_{j}(s, s)^{2} d s\right)\right]<\infty$ (the Novikov condition) which means that $\hat{P}$ and $Q$ are equivalent.

\subsection{Time homogeneity}

From the form of $H(u, x)$, and $X(t)=\left(X_{1}(t), \ldots, X_{n}(t)\right)^{\prime}$ we can see that the $P(t, T)$ are Markov and time homogeneous. As such the model plus knowledge of $X(t)$ gives us a set of theoretical prices which may differ from those observed. Under the original no-arbitrage framework (Flesaker \& Hughston, 1996) initial observed prices form part of the input (hence the earlier definition of $\phi(s)$ in equation (2.3)) but this results in the loss of time homogeneity. Both approaches have their own merits. Here the intention is that the number of factors, $n$, should be large enough to ensure that once the $X_{i}(t)$ have been estimated there is a close correspondence (but probably not exact) between theoretical and observed prices for all $t$. It can then be argued that frictions in the market such as transaction costs and buying and selling spreads prevent exploitation of the price errors. In the context of shortterm derivative pricing the $\mathrm{FH}$ approach to $\phi(s)$ is more popular with practitioners despite the obvious criticism that frequent recalibration of $\phi(s)$ (that is, treating $\phi(s) \equiv \phi(t, s)$ as stochastic) is inconsistent with the model assumption that $\phi(s)$ is deterministic.

\subsection{Practical considerations}

The structure of this model is such that only a limited number of random factors $\left(X_{i}(t)\right.$ for $\left.i=1, \ldots, n\right)$ need to be recorded in order for us to be able to reconstruct the evolution of the term structure through time, calculate prices, returns on assets and so on. From the computational point of view this offers an advantage over some models (for example, some of those based upon the Heath-Jarrow-Morton (1992) 
framework) which require a record of the entire forward rate curve at all times. This advantage is, of course, shared with many other models but it is important to record this fact. The time homogeneity of the model also results in computational gains, although mainly in the programming phase.

It is necessary to carry out numerical integration in order to compute bond prices and interest rates on a given date and given $\tilde{X}_{1}(t)$ (see equation 2.8). However, this step can be done in a straightforward and accurate way, since it only involves one-dimensional integration even for the multifactor version of the model.

An investigation of the properties of model reveals that the inclusion of correlation (the $\rho_{i j}$ ) between the $d X_{i}(t)$ does not significantly affect the range of possible yields curves on a given date. Consequently the quality of fit on any given date will not be substantially enhanced. It is, therefore, only when we consider the dynamics of the model when the correlations come into play. First, when we analyse historical data, the estimated values of the $d X_{i}(t)$ may (and, indeed, do) exhibit cross-correlation. Second, such correlations will affect the future distribution of $X(T)$ and therefore, for example, shift the balance of probabilities between certain shapes of yield curve. This final point is particularly important when we are considering derivative pricing as in equation (2.2).

The $X_{i}(t)$, for $i=1, \ldots, n$, follow standard, correlated Ornstein-Uhlenbeck processes under $\hat{P}$ (in particular, $X(t)$ is a multivariate Gaussian process under $\hat{P}$ ). It follows that, for low dimensional models (for example, $n=1$ or 2 ), straightforward and accurate numerical computations are possible for, for example, European and Bermudan-style derivative prices. Equally, for higher dimensional models, the multivariate normality of $X(t)$ makes the process particularly simple to simulate accurately under $\hat{P}$. The nature of the changes of measure for each of the $X_{i}(t)$ means that the $X_{i}(s)$ given $X_{i}(t)$ are no longer normally distributed under $Q$, making pricing under $\hat{P}$ much more attractive. For risk management purposes, we are often interested in the real-world measure $P$. If a constant market price of risk is employed relative to $Q$ then the same problem exists (that $X_{i}(s)$ is not normally distributed although over a one-month period the normal approximation is reasonable). As an alternative we can employ (as we propose in Section 4) a constant change of drift between $\hat{Z}(t)$ and $Z(t)$ (the $P$-Brownian motion). This ensures that the $X_{i}(t)$ still follow Ornstein-Uhlenbeck processes under $P$ (now with non-zero means). A less desirable consequence of this, though, is that this does occasionally allow risk-premia to become negative from time to time. The frequency of this clearly depends upon the parametrisation of the model and the size of the change of measure with a low frequency being tolerable for the sake of ease of simulation of the $X_{i}(t)$. 


\section{Properties of the one-factor model}

We will now look in more detail at the properties of the one-factor model.

Let us first define the functions

$$
\begin{aligned}
I_{k}(x) & =\int_{0}^{\infty} H(u, x) \sigma^{k} e^{-k \alpha u} d u \\
\text { where } H(u, x) & =\exp \left(-\beta u+\sigma x e^{-\alpha u}-\frac{\sigma^{2}}{4 \alpha} e^{-2 \alpha u}\right)
\end{aligned}
$$

for $k=0,1, \ldots$. Then we have

$$
\begin{aligned}
r(t) \equiv r(X(t)) & =\frac{H(0, X(t))}{\int_{0}^{\infty} H(u, X(t)) d u}=\frac{H(0, X(t))}{I_{0}(X(t))} \\
\text { and } V(t, t) & =\frac{\int_{0}^{\infty} H(u, X(t)) \sigma e^{-\alpha u} d u}{\int_{0}^{\infty} H(u, X(t)) d u}=\frac{I_{1}(X(t))}{I_{0}(X(t))} .
\end{aligned}
$$

By application of Ito's lemma it is straightforward to show that

$$
\begin{aligned}
& d r(t)=\hat{m}(X(t)) d t+\hat{s}(X(t)) d \hat{Z}(t) \text { under } \hat{P} \\
& \text { or } \quad d r(t)=\tilde{m}(X(t)) d t+\tilde{s}(X(t)) d \tilde{Z}(t) \quad \text { under } Q \\
& \text { where } \hat{s}(x)=\tilde{s}(x)=r(x)\left(\sigma-\frac{I_{1}(x)}{I_{0}(x)}\right) \\
& \hat{m}(x)=r(x)\left[-\alpha \sigma x+\frac{1}{2} \sigma^{2}+\alpha x \frac{I_{1}(x)}{I_{0}(x)}-\frac{1}{2} \frac{I_{2}(x)}{I_{0}(x)}+\frac{I_{1}(x)^{2}}{I_{0}(x)^{2}}-\sigma \frac{I_{1}(x)}{I_{0}(x)}\right]
\end{aligned}
$$

and $\tilde{m}(x)=\hat{m}(x)+V(t, t) \hat{s}(x)$

$$
=r(x)\left[-\alpha \sigma x+\frac{1}{2} \sigma^{2}+\alpha x \frac{I_{1}(x)}{I_{0}(x)}-\frac{1}{2} \frac{I_{2}(x)}{I_{0}(x)}\right] .
$$

We will now consider how the dynamics of $r(t)$ depend on $X(t)$ when $X(t)$ tends to its extremes at $-\infty$ and $+\infty$. Although this means considering extremely rare events, the results allow us to compare the model with others which have known properties. This permits us then to "guess" at certain properties of the new model which depend on tail events.

Theorem $3.1(x \rightarrow-\infty)$

(a)

$$
I_{k}(x) \sim\left(\frac{-1}{\sigma x}\right)^{k+\frac{\beta}{\alpha}+1} \frac{\sigma^{k}}{\alpha} \Gamma\left(k+\frac{\beta}{\alpha}\right) \quad \text { as } x \rightarrow-\infty
$$

where $\Gamma(r)=\int_{0}^{\infty} v^{r-1} e^{-v} d v$ is the standard Gamma function. 
(b)

$$
\frac{I_{k}(x)}{I_{0}(x)} \sim\left(\frac{-1}{\sigma x}\right)^{k}\left(\frac{\beta}{\alpha}+k-1\right) \times \ldots \times\left(\frac{\beta}{\alpha}+1\right) \times \frac{\beta}{\alpha} \quad \text { as } x \rightarrow-\infty .
$$

(c) For $X(t)$ large and negative the dynamics of $r(t)$ under $\hat{P}$ and under $\hat{Q}$ are approximately those of the log-normal process, that is

$$
\begin{aligned}
\hat{m}(x) & \sim-\alpha r(x) \log r(x) \quad \text { as } x \rightarrow-\infty \\
\tilde{m}(x) & \sim-\alpha r(x) \log r(x) \quad \text { as } x \rightarrow-\infty \\
\text { and } \hat{s}(x)=\tilde{s}(x) & \sim \sigma r(x) \quad \text { as } x \rightarrow-\infty .
\end{aligned}
$$

\section{Proof}

(a) By substitution of $\exp (-\alpha u)=v$ we have

$$
I_{k}(x)=\frac{\sigma^{k}}{\alpha} \int_{0}^{1} v^{k+\frac{\beta}{\alpha}-1} \exp \left[\sigma x v-\frac{\sigma^{2}}{4 \alpha} v^{2}\right] d v .
$$

Now do some further substitutions: $y=-x$ and $w=\sigma y v$. Hence

$$
\begin{aligned}
I_{k}(-y) & =\frac{\sigma^{k}}{\alpha(\sigma y)^{k+\frac{\beta}{\alpha}}} \int_{0}^{\sigma y} w^{k+\frac{\beta}{\alpha}-1} \exp \left[-w-\frac{w^{2}}{4 \alpha y^{2}}\right] d w \\
\Rightarrow \alpha y^{k+\frac{\beta}{\alpha}} \sigma^{\frac{\beta}{\alpha}} I_{k}(-y) & \rightarrow \int_{0}^{\infty} w^{k+\frac{\beta}{\alpha}-1} e^{-w} d w \text { as } y \rightarrow+\infty \\
& =\Gamma\left(k+\frac{\beta}{\alpha}\right) \\
\Rightarrow I_{k}(x) & \sim \frac{\Gamma\left(k+\frac{\beta}{\alpha}\right)}{\alpha \sigma^{\beta / \alpha}(-x)^{k+\beta / \alpha}} \text { as } x \rightarrow-\infty .
\end{aligned}
$$

(b) Hence

$$
\begin{aligned}
\frac{I_{k}(x)}{I_{0}(x)} & \sim \frac{\alpha \sigma^{\beta / \alpha}(-x)^{\beta / \alpha}}{\alpha \sigma^{\beta / \alpha}(-x)^{k+\beta / \alpha}} \times \frac{\Gamma\left(k+\frac{\beta}{\alpha}\right)}{\Gamma\left(\frac{\beta}{\alpha}\right)} \\
& =\frac{1}{(-x)^{k}} \prod_{i=0}^{k-1}\left(\frac{\beta}{\alpha}+i\right) \quad \text { as } x \rightarrow-\infty
\end{aligned}
$$

since $\Gamma(z+1)=z \Gamma(z)$ for all $z \geq 1$.

(c) Now recall that $r(x)=H(0, x) / I_{0}(x)=\exp \left(\sigma x-\sigma^{2} / 4 \alpha\right) / I_{0}(x)$. It follows that

$$
\begin{aligned}
\log r(x) & =\sigma x-\frac{\sigma^{2}}{4 \alpha}-\log I_{0}(x) \\
& \sim \sigma x \quad \text { as } x \rightarrow-\infty
\end{aligned}
$$


since $x^{-1} \log I_{0}(x) \rightarrow 0$ as $x \rightarrow-\infty$.

First consider the volatility of $r(X(t))$ as $X(t) \rightarrow-\infty$. From (b) we know that $I_{1}(x) / I_{0}(x) \rightarrow 0$ as $x \rightarrow-\infty$. It follows that $\hat{s}(x) / r(x)=\tilde{s}(x) / r(x) \rightarrow \sigma$ as $x \rightarrow-\infty$.

Second consider the drift of $r(X(t))$ as $X(t) \rightarrow-\infty$. Since $\sigma x \sim \log r(x)$ as $x \rightarrow-\infty$ the dominant term in both $\hat{m}(x)$ and $\tilde{m}(x)$ is $-\alpha r(x) \log r(x)$ as $x \rightarrow-\infty$. That is, $\hat{m}(x) \sim-\alpha r(x) \log r(x)$ and $\tilde{m}(x) \sim-\alpha r(x) \log r(x)$ as $x \rightarrow-\infty$.

It follows that for values of $r(t)$ very close to zero the dynamics of $r(t)$ are similar to those of the Black \& Karasinski (1991) model.

Theorem $3.2(x \rightarrow+\infty)$

(a)

$$
I_{k}(x) \sim \frac{\sigma^{k-1} e^{\sigma x-\sigma^{2} / 4 \alpha}}{\alpha x} \text { as } x \rightarrow+\infty .
$$

(b)

$$
\frac{I_{k}(x)}{I_{0}(x)} \rightarrow \sigma^{k} \text { as } x \rightarrow+\infty
$$

(c)

$$
I_{1}(x)-\sigma I_{0}(x) \sim \frac{-e^{\sigma x-\sigma^{2} / 4 \alpha}}{\alpha \sigma x^{2}} \text { as } x \rightarrow+\infty .
$$

(d)

$$
\sigma^{2} I_{0}(x)-I_{2}(x) \sim 2 \frac{e^{\sigma x-\sigma^{2} / 4 \alpha}}{\alpha x^{2}} \text { as } x \rightarrow+\infty .
$$

(e) For $X(t)$ large and positive the dynamics of $r(t)$ under $P$ and $Q$ are approximately those a of a Gaussian (Vasicek) process, that is

$$
\begin{aligned}
\frac{\hat{m}(x)}{r(x)} & \rightarrow-\alpha \text { as } x \rightarrow+\infty \\
\frac{\tilde{m}(x)}{r(x)} & \rightarrow-\alpha \text { as } x \rightarrow+\infty \\
\hat{s}(x) & \rightarrow \sigma \text { as } x \rightarrow+\infty \\
\text { and } \tilde{s}(x) & \rightarrow \sigma \text { as } x \rightarrow+\infty
\end{aligned}
$$

\section{Proof}


(a) Use the substitution $v=\sigma x\left(1-e^{-\alpha u}\right)$. Then we have

$$
I_{k}(x)=\frac{\sigma^{k-1} e^{\sigma x}}{\alpha x} \int_{0}^{\sigma x}\left(1-\frac{v}{\sigma x}\right)^{\frac{\beta}{\alpha}+k-1} \exp \left[-v-\frac{\sigma^{2}}{4 \alpha}\left(1-\frac{v}{\sigma x}\right)^{2}\right] d v .
$$

Now as $x \rightarrow \infty$

$$
\int_{0}^{\sigma x}\left(1-\frac{v}{\sigma x}\right)^{\frac{\beta}{\alpha}+k-1} \exp \left[-v-\frac{\sigma^{2}}{4 \alpha}\left(1-\frac{v}{\sigma x}\right)^{2}\right] d v \rightarrow \int_{0}^{\infty} e^{-v-\sigma^{2} / 4 \alpha} d v=e^{-\sigma^{2} / 4 \alpha} .
$$

Hence

$$
I_{k}(x) \sim \frac{\sigma^{k-1} e^{\sigma x-\sigma^{2} / 4 \alpha}}{\alpha x} \text { as } x \rightarrow \infty
$$

(b) It follows trivially that

$$
\frac{I_{k}(x)}{I_{0}(x)} \rightarrow \sigma^{k} \quad \text { as } x \rightarrow \infty
$$

We now note that (by reorganisation of equation (3.4)) that

$$
\hat{m}(x)=r(x)\left[\frac{\left(I_{1}(x)-\sigma I_{0}(x)\right)}{I_{0}(x)}\left(\alpha x+\frac{I_{1}(x)}{I_{0}(x)}\right)+\frac{1}{2} \frac{\left(\sigma^{2} I_{0}(x)-I_{2}(x)\right)}{I_{0}(x)}\right] .
$$

From equation (3.7) we observe that $\left(I_{k}(x)-\sigma^{k} I_{0}(x)\right) / I_{0}(x) \rightarrow 0$ as $x \rightarrow \infty$. It follows that it is appropriate to investigate the asymptotics of $I_{k}(x)-\sigma^{k} I_{0}(x)$.

(c)

$$
\begin{aligned}
I_{1}(x)-\sigma I_{0}(x) & =\frac{e^{\sigma x}}{\alpha \sigma x} \int_{0}^{\sigma x}\left(\frac{-v}{x}\right)\left(1-\frac{v}{\sigma x}\right)^{\frac{\beta}{\alpha}-1} \exp \left[-v-\frac{\sigma^{2}}{4 \alpha}\left(1-\frac{v}{\sigma x}\right)^{2}\right] d v \\
& \sim-\frac{e^{\sigma x-\sigma^{2} / 4 \alpha}}{\alpha \sigma x^{2}} \text { as } x \rightarrow \infty .
\end{aligned}
$$

(d) Similarly

$$
\begin{aligned}
I_{2}(x)-\sigma^{2} I_{0}(x) & =\frac{e^{\sigma x}}{\alpha \sigma x} \int_{0}^{\sigma x}\left(-\frac{2 \sigma v}{x}+\frac{v^{2}}{x^{2}}\right)\left(1-\frac{v}{\sigma x}\right)^{\frac{\beta}{\alpha}-1} \exp \left[-v-\frac{\sigma^{2}}{4 \alpha}\left(1-\frac{v}{\sigma x}\right)^{2}\right] d v \\
& \sim-\frac{2 e^{\sigma x-\sigma^{2} / 4 \alpha}}{\alpha x^{2}} \text { as } x \rightarrow \infty .
\end{aligned}
$$

(e) From (a) to (d) we can infer that

$$
\begin{aligned}
& \frac{x\left(I_{1}(x)-\sigma I_{0}(x)\right)}{I_{0}(x)} \rightarrow-1 \\
& \frac{x\left(I_{2}(x)-\sigma^{2} I_{0}(x)\right)}{I_{0}(x)} \rightarrow-2 \sigma \quad \text { as } x \rightarrow \infty .
\end{aligned}
$$


Combining these asymptotics with equation (3.8), it follows that $\hat{m}(x) / r(x) \rightarrow-\alpha$ as $x \rightarrow \infty$.

Also

$$
\frac{\tilde{m}(x)}{r(x)}=\frac{\hat{m}(x)}{r(x)}-\frac{I_{1}(x)}{I_{0}(x)}\left(\frac{I_{1}(x)-\sigma I_{0}(x)}{I_{0}(x)}\right) .
$$

But $I_{1}(x) / I_{0}(x) \rightarrow \sigma$ and $\left(I_{1}(x)-\sigma I_{0}(x)\right) / I_{0}(x) \rightarrow 0$ as $x \rightarrow \infty$. Thus $\tilde{m}(x) / r(x) \rightarrow$ $-\alpha$ as $x \rightarrow \infty$.

Now recall that

$$
r(x)=\frac{H(0, x)}{I_{0}(x)}=\frac{e^{\sigma x-\sigma^{2} / 4 \alpha}}{I_{0}(x)}, \quad \Rightarrow r(x) \sim \alpha \sigma x \quad \text { as } x \rightarrow \infty .
$$

Thus

$$
\hat{s}(x)=\tilde{s}(x)=r(x)\left(\frac{\sigma I_{0}(x)-I_{1}(x)}{I_{0}(x)}\right) \rightarrow \alpha \sigma \quad \text { as } x \rightarrow \infty .
$$

So we can see that at its extremes the one-factor model can act like the Black \& Karasinski (1991) model when $r(t)$ is very small and like the Vasicek (1977) model when $r(t)$ is very large. The latter observation suggests that the present model does not share the problem that the Black \& Karasinski model has with infinite futures prices (see, for example, Hunt \& Kennedy, 2000, page 322) and infinite expected values for the accumulated value of a cash investment (see, for example, James \& Webber, 2000, page 231).

\section{Multifactor models}

In this section we will discuss a variety of aspects of the model including qualitative issues relating to calibration of the model parameters. In particular, we aim to establish that the new family of models is very suitable for a variety of applications including long-term risk management.

We will argue that the $\alpha_{i}$ are central to the validity of the family of multifactor models. In particular, the smallest of the $\alpha_{i}$ serves two key purposes. First, it allows long-dated bond yields to take a wide range of values with reasonable probability. Second, a small value of $\alpha_{i}$ means that $X_{i}(t)$ reverts very slowly. The consequent long cycles experienced by $X_{i}(t)$ feed through to sustained periods of both high and low interest rates. Both of these characteristics can be clearly observed in historical data. For example, in Figure 1.1 we can see that long-dated bond yields have, over a period of 100 years ranged from below $2.5 \%$ to over $15 \%$. Additionally, in many countries there have been periods of several decades when interest rates were low and stable and other sustained periods when interest rates were high and more volatile. 
In the numerical examples that follow we will concentrate on the two-factor version of the model. This allows us to visualise certain features of the multifactor version of the model through the use of contour plots. However, it should be borne in mind that other analyses (for example, Cairns, 1998, Feldman et al., 1998, Rebonato, 1998) suggest that three or more factors might be appropriate.

\subsection{Yield curves}

A wide variety of yield curve shapes can be generated depending upon the number of factors. As an example consider the two-factor model with

$$
\alpha_{1}=0.6, \quad \alpha_{2}=0.06, \quad \sigma_{1}=0.6, \quad \sigma_{2}=0.4, \quad \rho_{12}=-0.5, \quad \beta=0.04 .
$$

In Figure 4.1 we can see typical spot-rate curves (that is, $R(t, T)=-(T-t)^{-1} \log P(t, T)$ ) for this model and parameter set. Thus we have the classic rising and falling yield curves along with humped, dipped and flat curves. Of particular note, perhaps, is curve F. This starts and remains close to 0 for some time before gradually picking up. This sort of curve is similar to that which has been typical in Japan for some time (2002). This is a feature which some other positive-interest models cannot produce, such as the positive affine term-structure models. The key to this type of behaviour was indirectly highlighted in our analysis of the one factor model, where, as $r(t)$ gets small both its drift and volatility decline to zero. Besides the present model it is similar to the Black-Karasinski model.

We can also observe from Figure 4.1 that even though all of the spot-rate curves converge ultimately to $4 \%(\beta)$ they still show considerable diversity at $T-t=30$ years. This is a direct consequence of the low choice of $\alpha_{2}=0.06$. The smaller this value is the greater the variation we see in spot rates and other rates of interest at long maturity dates. Long-dated par yields tend to show up a greater degree of variation, and, indeed, do not converge to the same value. This amount of variation is exactly what we observe in historical data (for example, see Figures 1.1 and 1.3).

In Figure 4.2 we give contour plots for the risk-free rate, $r(t)$, the 20-year spot and forward rates, $R(t, t+20)$ and $f(t, t+20)$, and the par yield on consols (irredeemable bonds), $\rho(t)$. In each plot, the ellipse centred on $(0,0)$ gives the $90 \%$ and $99 \%$ unconditional confidence regions for $X(t)$ under $\hat{P}$. This figure tells us how the four rates of interest depend on $X_{1}(t)$ and $X_{2}(t)$. Thus we can see that $f(t, t+20)$ depends almost entirely on $X_{2}(t)$. In contrast, $r(t)$ is most dependent upon $X_{1}(t)$ although it also depends to some extent on $X_{2}(t)$. In between these two we can see that $R(t, t+20)$ and $\rho(t)$ are primarily determined by $X_{2}(t)$ but have a small dependence on $X_{1}(t)$. 


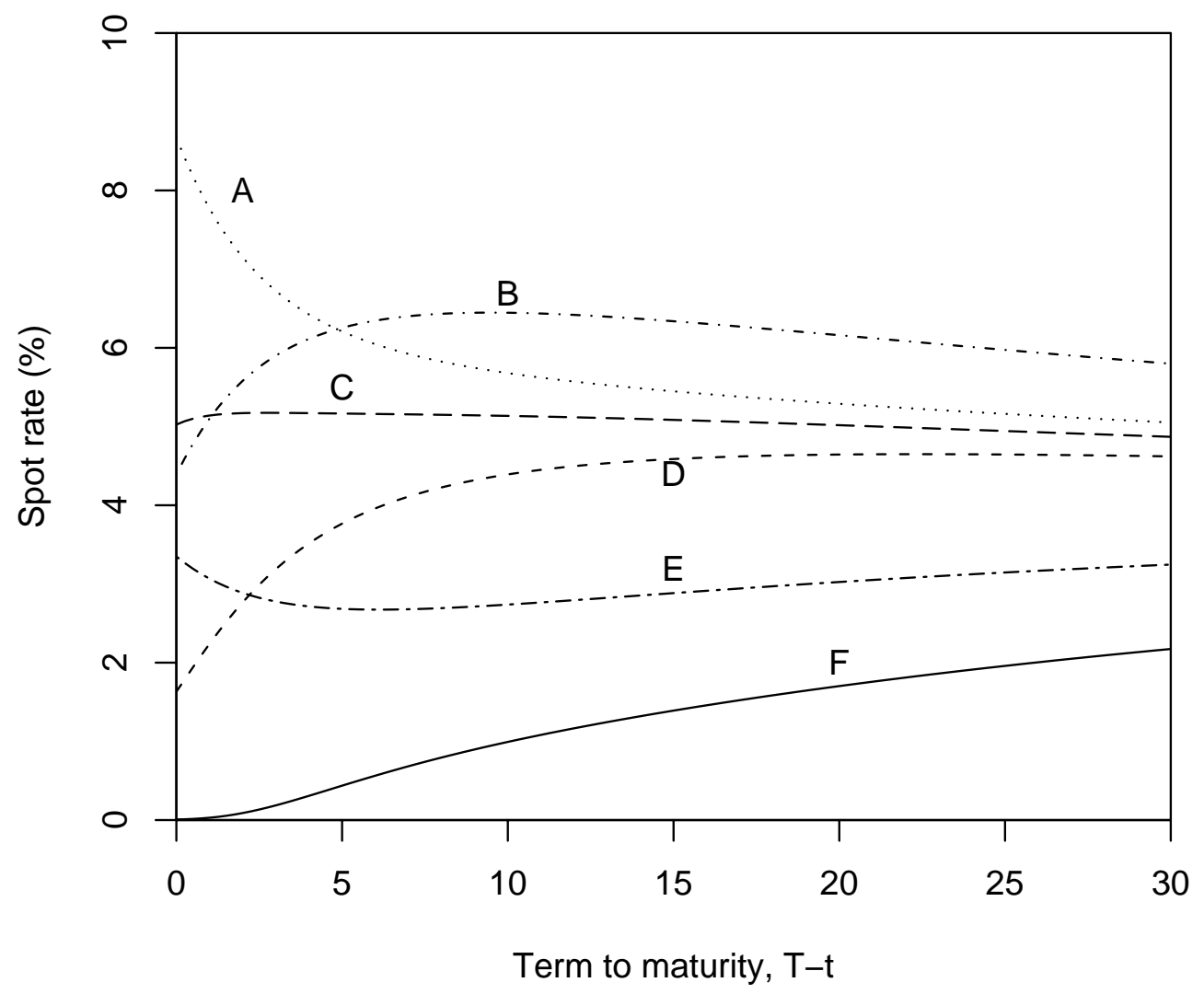

Figure 4.1: Sample spot-rate curves, $R(t, T)$, for the two-factor model. $\alpha=$ $(0.6,0.06)^{\prime}, \sigma=(0.6,0.4)^{\prime}, \rho_{12}=-0.5, \beta=0.04 . \quad \mathrm{A}: X(t)=(1,3)^{\prime} ; \mathrm{B}:$ $X(t)=(-1,5)^{\prime} ; \mathrm{C}: X(t)=(0,3)^{\prime} ; \mathrm{D}: X(t)=(-2,3)^{\prime} ; \mathrm{E}: X(t)=(1,-1)^{\prime} ; \mathrm{F}:$ $X(t)=(-8,-4)^{\prime}$. 

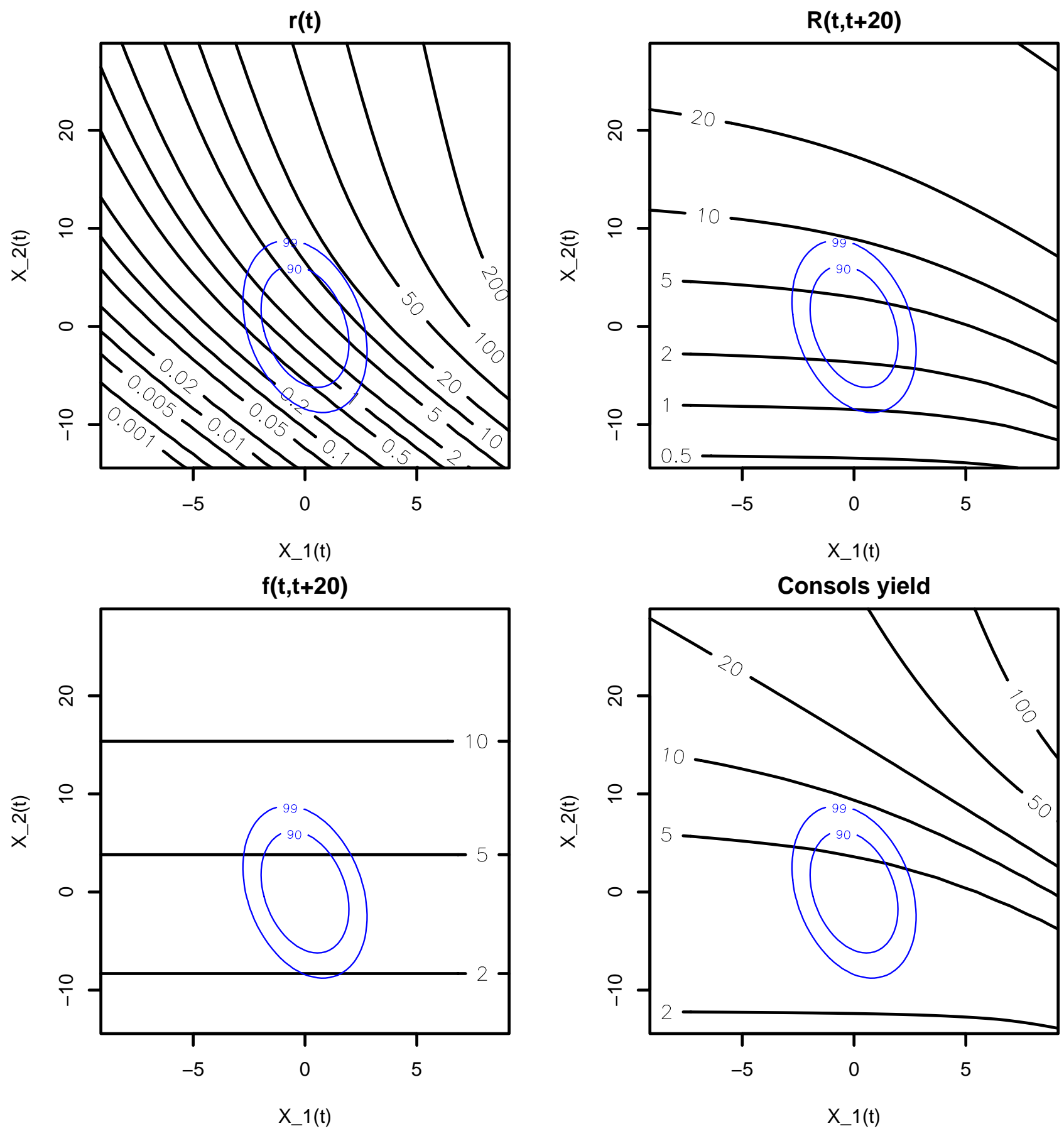

Figure 4.2: Dependence of different interest rates on $X_{1}(t)$ and $X_{2}(t)$ for the parameters $\alpha=(0.6,0.06)^{\prime}, \sigma=(0.6,0.4)^{\prime}, \rho_{12}=-0.5, \beta=0.04$. Interest rates considered are: the risk-free rate, $r(t)$; the 20-year spot rate, $R(t, t+20)$; the 20-year forward rate, $f(t, t+20)$; and the par yield on consols (irredeemable bonds), $\rho(t)$. Each graph is a contour plot with lines connecting pairs of points $\left(X_{1}(t), X_{2}(t)\right)$ with the same rate of interest. The thin ellipses give confidence regions for $X(t)$ under $\hat{P}$. 


\subsection{Short and long-term variability: approximations}

Recall that $f(t, T)(X(t))=H(T-t, X(t)) / \int_{T-t}^{\infty} H(u, X(t)) d u$ and the consols yield is $\rho(t)(X(t))=\int_{0}^{\infty} H(u, X(t)) d u / \int_{0}^{\infty} u H(u, X(t)) d u$. Without loss of generality suppose that $t=0$ and define

$$
\begin{aligned}
I_{0}(T, x) & =\int_{T}^{\infty} H(u, x) d u \\
\text { and } I_{1 i}(T, x) & =\frac{\partial I_{0}}{\partial x_{i}}(T, x)=\int_{T}^{\infty} \sigma_{i} e^{-\alpha_{i} u} H(u, x) d u
\end{aligned}
$$

Also note that

$$
\frac{\partial H}{\partial x_{i}}(T, x)=\sigma_{i} e^{-\alpha_{i} T} H(T, x)
$$

Then

$$
\begin{aligned}
\frac{\partial f}{\partial x_{i}}(0, T)(x) & =I_{0}(T, x)^{-2}\left[\frac{\partial H}{\partial x_{i}}(T, x) I_{0}(T, x)-H(T, x) \frac{\partial I_{0}}{\partial x_{i}}(T, x)\right] \\
& =f(0, T)(x) \sigma_{i} e^{-\alpha_{i} T}-f(0, T)(x) \frac{I_{1 i}(T, x)}{I_{0}(T, x)}
\end{aligned}
$$

Now we will make a very crude approximation by ignoring the $\rho_{i j} \sigma_{i} \sigma_{j}$ terms in $H(T, x), I_{0}(T, x)$ and $I_{1 i}(T, x)$. Thus, for $x=0$

$$
\begin{aligned}
H(T, 0) & \approx e^{-\beta T} \\
I_{0}(T, x) & \approx \int_{T}^{\infty} e^{-\beta u} d u=\frac{e^{-\beta T}}{\beta} \\
I_{1 i}(T, x) & \approx \int_{T}^{\infty} \sigma_{i} e^{-\alpha_{i} u} e^{-\beta u} d u=\frac{\sigma_{i} e^{-\left(\beta+\alpha_{i}\right) T}}{\beta+\alpha_{i}} .
\end{aligned}
$$

It follows that

$$
\begin{aligned}
\frac{\partial f}{\partial x_{i}}(0, T)(0) & \approx f(0, T)\left[\sigma_{i} e^{-\alpha_{i} T}-\frac{\sigma_{i} e^{-\left(\beta+\alpha_{i}\right) T} /\left(\beta+\alpha_{i}\right)}{e^{-\beta T} / \beta}\right] \\
& =f(0, T)(0) \frac{\alpha_{i} \sigma_{i} e^{-\alpha_{i} T}}{\beta+\alpha_{i}}
\end{aligned}
$$

If we apply the same approximation to $f(0, T)(0)$ we have $f(0, T)(0) \approx \beta$.

It follows that, as a very crude approximation,

$$
\begin{aligned}
f(t, T)(X(t)) & \approx \beta\left(1+\sum_{i=1}^{n} d_{i}(T-t) X_{i}(t)\right) \\
\text { where } d_{i}(\tau) & =\frac{\alpha_{i} \sigma_{i} e^{-\alpha_{i} \tau}}{\beta+\alpha_{i}} .
\end{aligned}
$$


Now recall that the local volatility of each $X_{i}(t)$ is 1 with correlations $\rho_{i j}$, so the local variance of $f(t, T)$ if $X(t) \approx 0$ will be approximately

$$
\beta^{2} \sum_{i, j=1}^{n} d_{i}(T-t) d_{j}(T-t) \rho_{i j} .
$$

The unconditional covariance of $X_{i}(t)$ and $X_{j}(t)$ is $\rho_{i j} /\left(\alpha_{i}+\alpha_{j}\right)$. It follows that the unconditional variance of $f(t, T)(X(t))$ will be approximately

$$
\beta^{2} \sum_{i, j=1}^{n} \frac{d_{i}(T-t) d_{j}(T-t) \rho_{i j}}{\alpha_{i}+\alpha_{j}}
$$

Suppose that $\alpha_{1}>\alpha_{2}>\ldots>\alpha_{n}>0$. The effect of the $1 /\left(\alpha_{i}+\alpha_{j}\right)$ components means that the process $X_{n}(t)$ (smallest $\alpha_{i}$ ) will have proportionately a greater effect on long-term variability that on short-term variability.

We can also see from the definitions of the $d_{i}(\tau)$ that the process $X_{n}(t)$ will have proportionately a greater effect on long-dated interest rates than on short-dated rates.

We can show in a similar fashion that the par yield on irredeemable bonds is approximately

$$
\begin{aligned}
\rho(t) & \approx \beta\left[1+\sum_{i=1}^{n} e_{i} X_{i}(t)\right] \\
\text { where } e_{i} & =\frac{\alpha_{i} \sigma_{i} \beta}{\left(\beta+\alpha_{i}\right)^{2}} .
\end{aligned}
$$

Hence its short-term variance will be approximately

$$
\beta^{2} \sum_{i, j=1}^{n} e_{i} e_{j} \rho_{i j}
$$

and its unconditional variance will be approximately

$$
\beta^{2} \sum_{i, j=1}^{n} \frac{e_{i} e_{j} \rho_{i j}}{\alpha_{i}+\alpha_{j}}
$$

As a final comment, refer back to equations (4.4) and (4.5). We can see from these equations that, at least as a first approximation, changes in the forward rate curve are made up of a combination of exponentials with rates $\alpha_{1}, \ldots, \alpha_{n}$. This means that we can generate over the short term the usual range of changes in the shape of the forward-rate curve such as changes in slope, humps and twists (depending upon how many factors there are. 
We have stressed that these are crude approximations. However, they are very useful as part of a subjective calibration of the model. For example, we can specify the level of variability in short and long-dated interest rates in both the short and the long term. These approximations can be used to get a first estimate of the parameter values which will achieve these aims.

\subsection{The volatility term structure}

We will now consider the full term structure of volatility.

Recall from equation (4.3) that the volatility of $f(t, T)$ with respect to changes in $X_{i}(t)$ is

$$
v_{i}(t, T)(x)=\frac{\partial f}{\partial x_{i}}(t, T)(x)=f(t, T)(x)\left[\sigma_{i} e^{-\alpha_{i}(T-t)}-\frac{I_{1 i}(T-t, x)}{I_{0}(T-t, x)}\right] .
$$

Now the $X_{i}(t)$ will be correlated, so that these volatility curves can be thought of as dependent. Recall that we defined the matrix $C$ so as to satisfy $C C^{\prime}=\left(\rho_{i j}\right)$. In particular, we now choose $C$ to be lower triangular (thus defining $C$ uniquely) resulting in, for each $i$

$$
d X_{i}(t)=-\alpha_{i} X_{i}(t) d t+\sum_{j=1}^{i} c_{i j} d \hat{Z}_{j}(t)
$$

We can then see that the independent volatility curve for $f(t, T)$ corresponding to $d \hat{Z}_{j}(t)$ is

$$
\tilde{v}_{j}(t, T)(x)=\sum_{i=j}^{n} c_{i j} v_{i}(t, T)(x) .
$$

We will now illustrate the volatility curves for the parameter set given earlier in equation (4.1). In Figure 4.3 we plot volatility curves for a typical rising yield curve (with a slight hump) and a fairly flat curve.

We can make the following empirical observations:

- Volatility is higher when interest rates are higher. This is merely a reflection of the common factor of $f(t, T)$ in equation (4.9).

- Changes in $X_{2}(t)$ have, proportionately, a greater effect on longer-dated forward rates. This backs up earlier observations.

- The independent volatility curves are, perhaps, more informative.

Typically, for the chosen decomposition for $C, \tilde{v}_{2}(t, T)$ provides us with a change in the slope or level of $f(t, T)$, while $\tilde{v}_{1}(t, T)(x)$ provides us with a twist or a hump. 

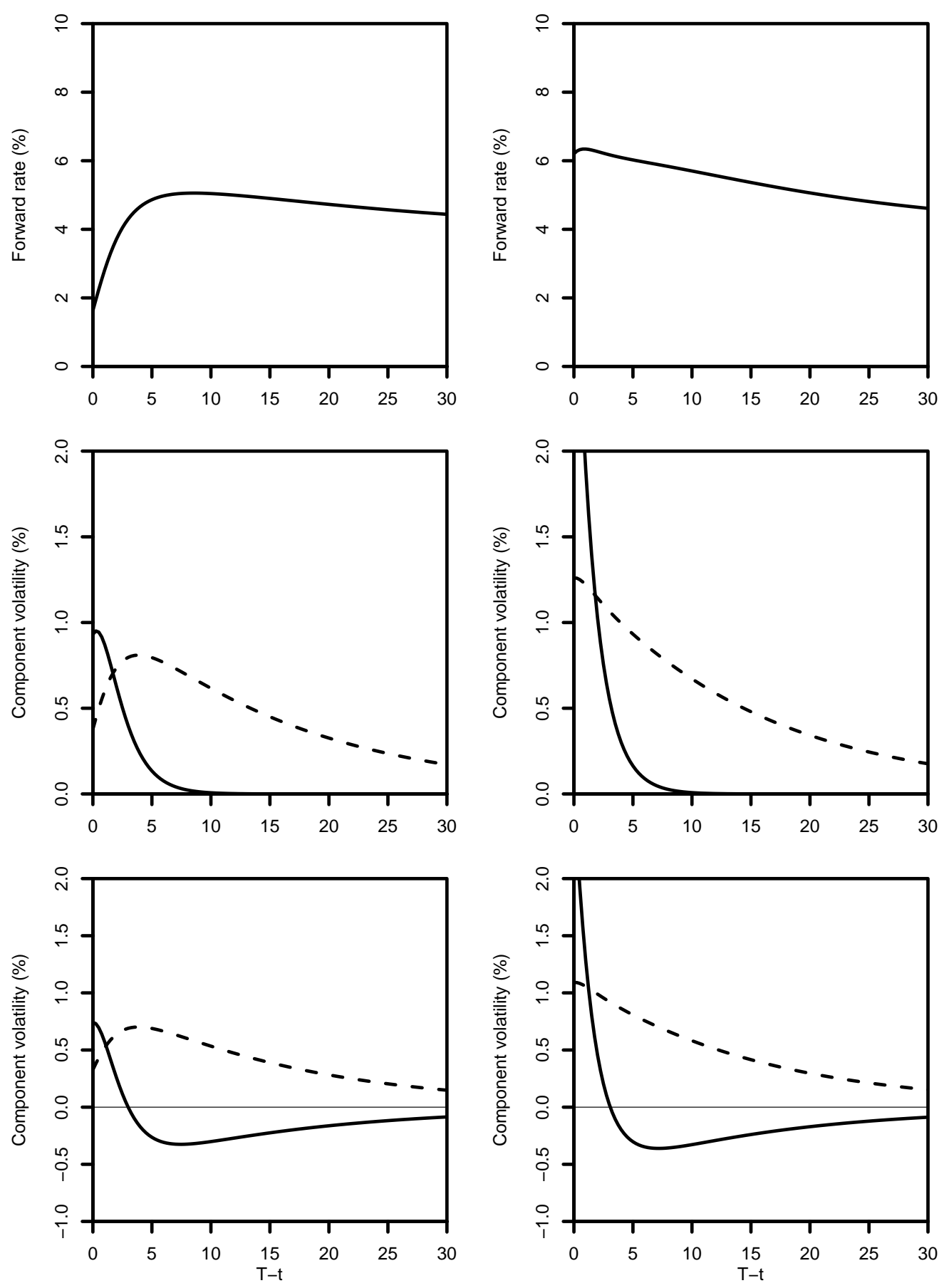

Figure 4.3: Sample forward-rate volatility curves for the two-factor model. $\alpha=$ $(0.6,0.06)^{\prime}, \sigma=(0.6,0.4)^{\prime}, \rho_{12}=-0.5, \beta=0.04$. Left-hand column: $X_{1}(t)=$ $-2, X_{2}(t)=3$. Right-hand column: $X_{1}(t)=0, X_{2}(t)=4$. Top row: forward-rate curve, $f(t, T)(X(t))$. Middle row: volatility with respect to short-term changes in $X_{1}(t)$, and $X_{2}(t)$ - solid curve $v_{1}(t, T)(X(t))$; dashed curve $v_{2}(t, T)(X(t))$. Bottom row: independent volatility curves - solid curve $\tilde{v}_{1}(t, T)(X(t))$; dashed curve $\tilde{v}_{2}(t, T)(X(t))$. 


\subsection{Bond volatilities}

In equation (2.7) we noted that the SDE for $P(t, T)$ is

where, here, we have

$$
d P(t, T)=P(t, T)\left[r(t) d t+S_{P}(t, T)^{\prime}(d \hat{Z}(t)-V(t, t) d t)\right]
$$

$$
\begin{aligned}
S_{P}(t, T) & =V(t, T)-V(t, t) \\
V_{j}(t, T) & =\frac{\int_{T-t}^{\infty} H(u, X(t)) \bar{\sigma}_{j}(u) d u}{\int_{T-t}^{\infty} H(u, X(t)) d u} \\
& =\frac{\sum_{i=1}^{n} c_{i j} I_{1 i}(T-t, X(t))}{I_{0}(T-t, X(t))} \\
\sum_{k=1}^{n} c_{i k} c_{j k} & =\rho_{i j} \\
\text { and } \bar{\sigma}_{j}(u) & =\sum_{i=1}^{n} c_{i j} \sigma_{i} e^{-\alpha_{i} u} .
\end{aligned}
$$

It follows that the overall squared volatility of $P(t, T)$ is

$$
\begin{aligned}
\sum_{j=1}^{n} S_{P j}(t, T)^{2} & =\sum_{j=1}^{n}\left(V_{j}(t, T)-V_{j}(t, t)\right)^{2} \\
& =\sum_{i, k=1}^{n} \rho_{i k} \Delta_{i}(t, T) \Delta_{k}(t, T) \\
\text { where } \Delta_{i}(t, T) & =\frac{I_{1 i}(T-t, X(t))}{I_{0}(T-t)}-\frac{I_{1 i}(0, X(t))}{I_{0}(0)} .
\end{aligned}
$$

As illustrative examples, the independent volatility curves $S_{P j}(t, T)$ for the same two cases as in the previous section are plotted in Figure 4.4.

We can see that tpyically the sign of the volatility curves is the opposite of what we observed in the corresponding forward-rate volatility curves (although the zerocoupon bond price volatilities are more closely linked to spot-rate volatilities).

\subsection{Real-world probabilities and risk premiums}

Earlier we defined the risk-neutral equivalent measure $Q$ by introducing the drift $V(t, t)$ : that is, under $Q$, the $d \tilde{Z}_{j}(t)=d \hat{Z}_{j}(t)-V_{j}(t, t) d t$ are independent standard Brownian motions. This leaves us with the usual dynamics for the $P(t, T)$ under $Q: d P(t, T)=P(t, T)\left[r(t) d t+S_{P}(t, T)^{\prime} d \tilde{Z}(t)\right]$. The nature of $V(t, t)$ means that there is no particular gain to be made from looking directly at $Q$ : pricing is much 

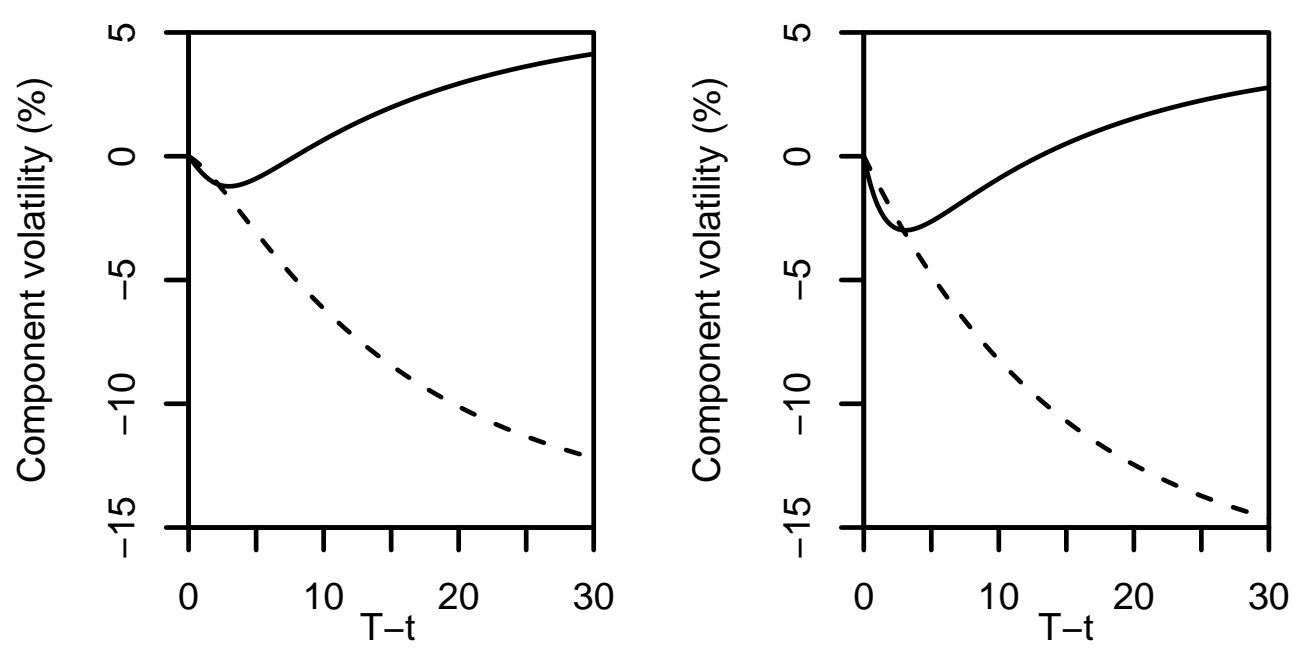

Figure 4.4: Sample zero-coupon price volatility volatility curves, $S_{P i}(t, T)$ for the two-factor model. Solid curves: $i=1$. Dashed curves: $i=2 . \quad \alpha=(0.6,0.06)^{\prime}$, $\sigma=(0.6,0.4)^{\prime}, \rho_{12}=-0.5, \beta=0.04$. Left: $X_{1}(t)=-2, X_{2}(t)=3$. Right: $X_{1}(t)=0, X_{2}(t)=4$.

easier under $\hat{P}$ rather than under $Q$ where the the $X_{i}(t)$ are no-longer normally distributed.

However, it is very much of interest and practical relevance to look at dynamics under the real-world measure $P$. In particular, if the model is to be used as a tool in long-term risk management (for example, in dynamic financial analysis or pension-plan asset allocation).

In order to do this we need to model the market prices of risk which take us from the risk-neutral measure, $Q$, to the real-world measure, $P$. We introduce appropriate drifts $\gamma_{j}(t)$ which satisfy the Novikov condition and define $Z(t)$ to be a standard Brownian motion under $P$ with $d Z(t)=d \tilde{Z}(t)+\gamma(t) d t=d \hat{Z}(t)-V(t, t) d t+\gamma(t) d t$.

As part of our modelling assumptions we propose that $\gamma(t)=V(t, t)+\theta$ for some constant vector $\theta$. This component of the model is a pragmatic choice which gives $d Z(t)=d \hat{Z}(t)+\theta d t$ and ensures that the $X_{i}(t)$ are normally distributed under $P$ as well as $\hat{P}$ but with non-zero means. Thus

$$
\begin{aligned}
& d X_{i}(t)=-\alpha_{i} X_{i}(t) d t+\sum_{j=1}^{n} c_{i j}\left(d Z_{j}(t)-\theta_{j} d t\right) \\
& =\alpha_{i}\left(\mu_{i}-X_{i}(t)\right) d t+\sum_{j=1}^{n} c_{i j} d Z_{j}(t) \\
& \text { where } \mu_{i}=-\alpha_{i}^{-1} \sum_{j=1}^{n} c_{i j} \theta_{j} \text {. }
\end{aligned}
$$


In practical terms it is useful to specify the $\mu_{i}$ first and then derive the $\theta_{j}$. Thus

$$
\theta=-C^{-1} \operatorname{diag}\left(\alpha_{i}\right) \mu
$$

where $\operatorname{diag}\left(\alpha_{i}\right)$ is the diagonal matrix with elements $\left(\alpha_{1}, \ldots, \alpha_{n}\right)$.

Let us now return to the $\mathrm{SDE}$ for $P(t, T)$.

$$
\begin{aligned}
d P(t, T) & =P(t, T)\left[r(t) d t+S_{P}(t, T)^{\prime}(d Z(t)-\gamma(t) d t]\right. \\
& =P(t, T)\left[\left(r(t)-S_{P}(t, T)^{\prime}(V(t, t)+\theta)\right) d t+S_{P}(t, T)^{\prime} d Z(t)\right] .
\end{aligned}
$$

It follows that the risk premium on $P(t, T)$ (that is, the excess expected return over the risk-free rate, $r(t))$ is

$$
-S_{P}(t, T)^{\prime}(V(t, t)+\theta) .
$$

Sample contour plots for risk premiums are given in Figure 4.5 for the 30-year zerocoupon bond. In this set of plots we aim to illustrate how risk premiums depend upon the parameter vector $\mu$ (equivalently, $\theta$ ).

- The case where $\mu=(-2,6)^{\prime}$ gives a reasonable outcome. By overlaying the unconditional confidence region for $X(t)$ we can see which values are most likely under $P$. Most of the time, then, the 30-year risk premium will be positive. However, from time to time, it will become negative corresponding to periods when interest rates are low. This is a plausible scenario. For example, in the UK in 2002 low interest rates have resulted in unprecedented demand for long-dated bonds from life insurers and pension plans in an attempt to protect guaranteed fixed liabilities.

Other aspects of this choice for $\mu$ are reasonable by considering the confidence region relative to lines $\mathrm{A}$ and $\mathrm{B}$. Most of the region lies to the left of line $\mathrm{A}$ indicating that the spot-rate curve is typically (but not always) rising from 0 to 20 years (or humped). Rising curves are what we expect to see, although this is, of course, connected to positive risk premiums. Most of the region also lies above line $\mathrm{B}$ which indicates that spot rates are typically falling between 20 and 30 years to maturity. This decline is a feature of the study by Brown $\&$ Schaefer (2000). This persistent decline at the long end of the yield curve reflects stronger demand for long-dated over medium-dated bonds under most interest-rate scenarios, primarily again from life-insurers and pension plans.

Other choices for $\mu$ have positive and negative aspects.

- $\mu=(-4,4)$ gives more positive risk premiums. On the other hand the 20-30 year section of the spot-rate curve is falling as often as it is rising. The nature 
of the model (in particular, the low value of $\alpha_{2}$ ) means that $X(t)$ could easily spend 50 years or more above the line $\mathrm{B}$, giving the impression that spot rates normally fall between 20 and 30 years. The $99 \%$ confidence region lies entirely to the left of line A meaning that $r(t)$ is almost always lower than $R(t, t+20)$. This seems unlikely.

- $\mu=(0,0)^{\prime}$ has mainly small positive risk premiums with rising spot rates from 20 to 30 years but a significant chance that $r(t)$ is higher than $R(t, t+20)$. In general, interest rates will be relatively low. (See, also, Figure 4.2.)

- $\mu=c(1,6)^{\prime}$ looks very poor. Risk premiums are all significantly negative and, connected to this, the yield curve is typically falling.

In Figure 4.6 we look at the term structure of the risk premium for our standard parameter set and $\mu=(-2,6)^{\prime}$. Two sets of values for $X(t)$ are given $(-2,0)^{\prime}$ and $(1,3)^{\prime}$. For reference the spot-rate curves, $R(t, T)$, are plotted (top left), so that we can see that we have one low and rising curve and one high and falling. In the top right-hand plot we can see how the risk premium (equation 4.10) varies with term to maturity. In general, for this parameter set and choice for $\mu$ we find that the risk-premium term structure tends to peak at around 5 years. We can also see that, as in Figure 4.5, the risk premium depends upon $X(t)$. We note also that, when interest rates are very low (particularly short-dated rates), the risk premium can become slightly negative as indicated in the earlier discussion. In the lower plots we can see the full dependence of the 5-year and 30-year zero-coupon bond risk premiums on $X_{1}(t)$ and $X_{2}(t)$. The pattern of dependence is similar but 5 -year risk premiums are consistently higher for this choice of parameters.

\subsection{Simulation}

In the previous subsection we proposed a particular type of transformation, as part of the model, to take us from $\hat{P}$ to the real-world measure $P$. This particular choice makes simulation of sample paths of $X(t)$ particularly simple, since we can exploit the known distributional properties of the correlated Ornstein-Uhlenbeck process to simulate exactly in discrete time.

As an example, a typical sample path is represented in Figure 4.7. Here we plot the risk-free rate, $r(t)$, and the consols rate, $\rho(t)$. Also plotted are the corresponding sample paths for $X_{1}(t)$ and $X_{2}(t)$.

We can note that $X_{2}(t)$ has a sustained peak at A and a sustained trough at $\mathrm{B}$ which are reflected in peaks and troughs in the two interest rates. We can clearly see the linkage between both interest rates and $X_{2}(t)$. Since $X_{2}(t)$, through the low value of $\alpha_{2}$, has long cycles, we see that both rates of interest are also subject to long cycles of both high and low values. This type of behaviour is similar to that 

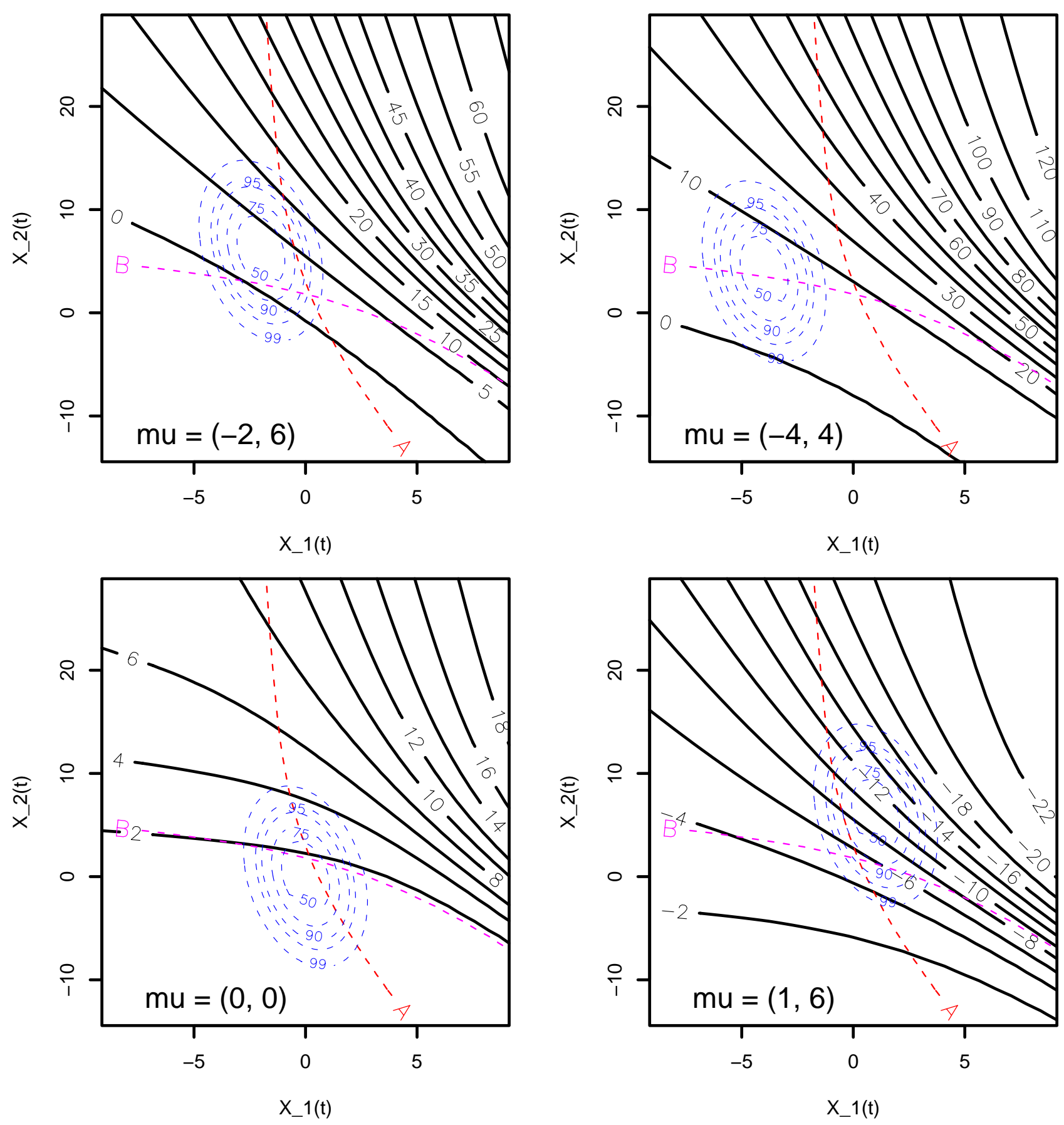

Figure 4.5: Risk premium on the 30-year zero-coupon bond as a function of the state variables $X_{1}(t)$ and $X_{2}(t)$ when $\alpha=(0.6,0.06)^{\prime}, \sigma=(0.6,0.4)^{\prime}, \rho_{12}=-0.5, \beta=0.04$. The four plots show how the risk premium depends upon the unconditional mean, $\mu$, of $X(t)$ under the real-world measure $P$. The thick solid lines give countours (in \%) of points with equal risk premiums. The thin solid lines give the $50 \%, 75 \%$, $90 \%, 95 \%$ and $99 \%$ confidence regions for $X(t)$ under $P$. Dashed lines A and B give an indication of the shape of the spot-rate curve. To the left (right) of A $r(t)-R(t, t+20)$ is positive (negative). Above (below) B $R(t, t+20)-R(t, t+30)$ is negative (positive). 


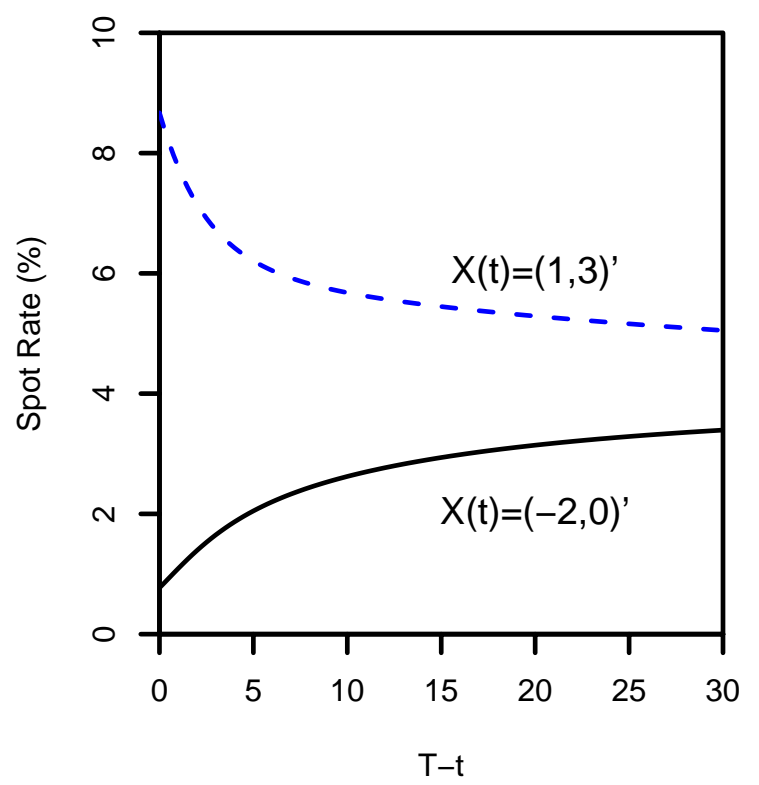

$P(t, t+5)$ risk premium

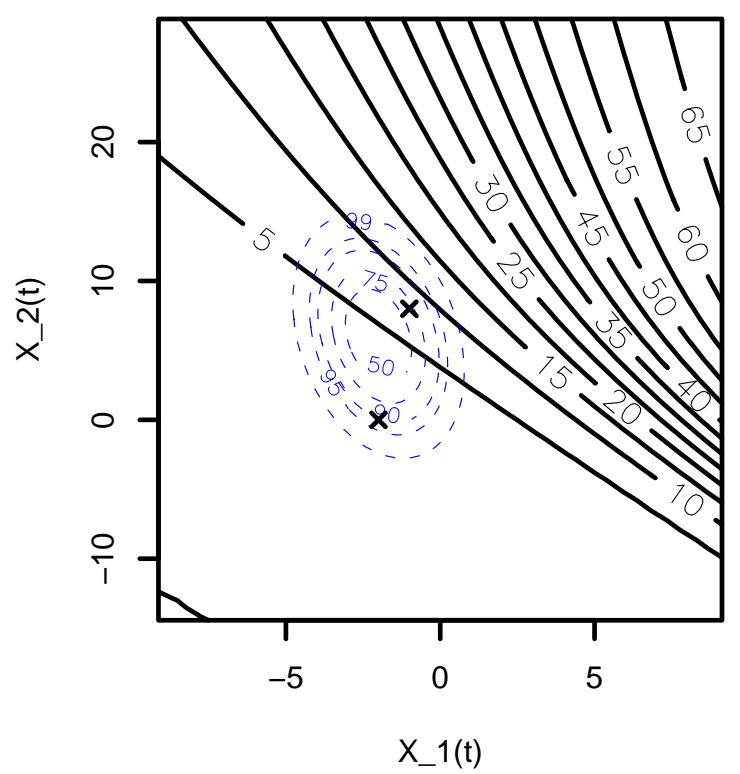

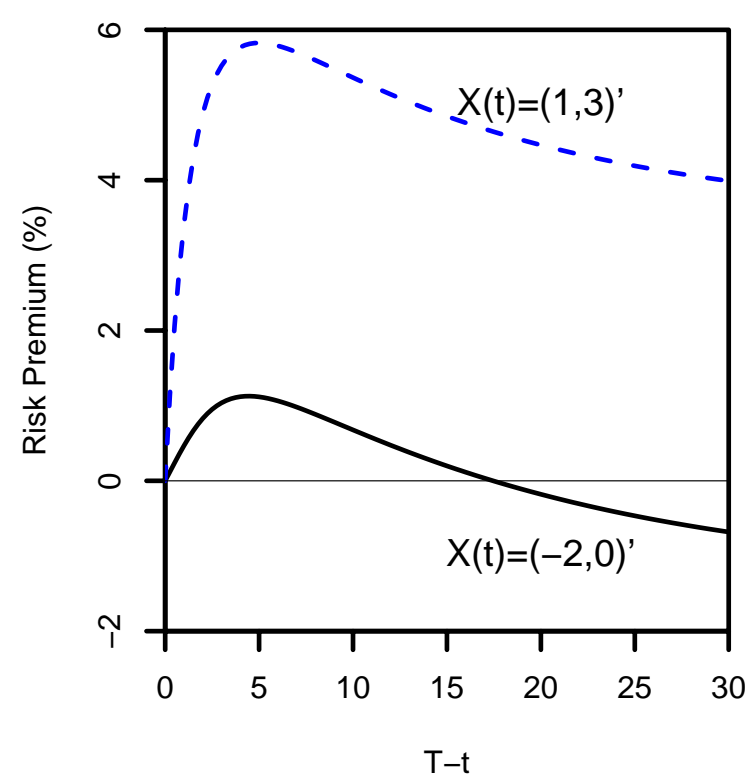

$\mathbf{P}(t, t+30)$ risk premium

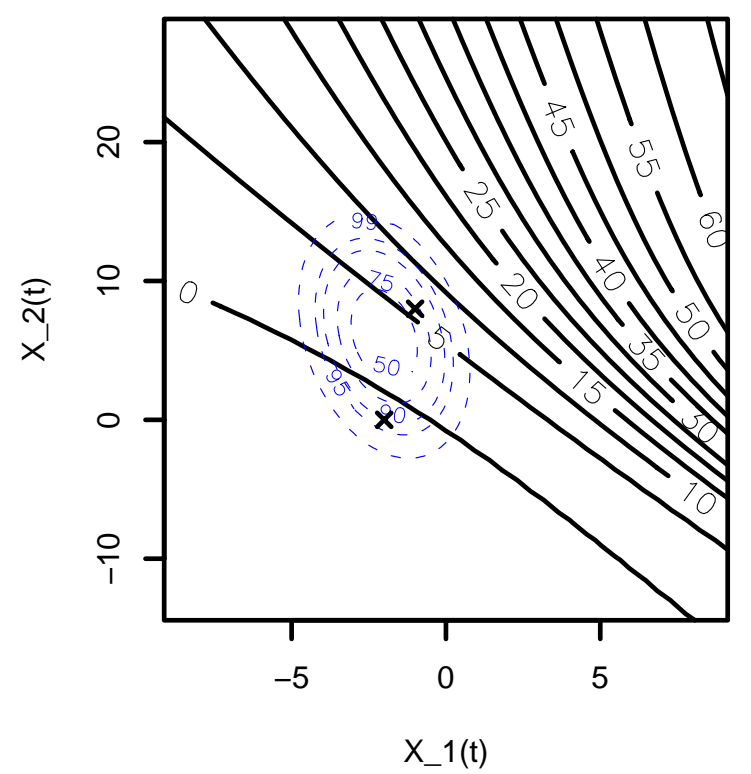

Figure 4.6: Risk premiums on zero-coupon bonds related to term to maturity. $\alpha=$ $(0.6,0.06)^{\prime}, \sigma=(0.6,0.4)^{\prime}, \rho_{12}=-0.5, \beta=0.04$ and $\mu=(-2,6)^{\prime}$. Top left: forwardrate curves for $X(t)=(-2,0)^{\prime}$ and $X(t)=(1,3)^{\prime}$. Top right: corresponding riskpremium term structure for zero-coupon bonds. Bottom left: contour plot of the risk premium for 5-year zero-coupon bonds. Bottom right: contour plot of the risk premium for 30-year zero-coupon bonds. (For a description of the contour plots, see Figure 4.5 caption.) The crosses mark the two locations of $X(t)$ illustrated. 
which we observed in both the UK and US data in Figures 1.1 and 1.3. We can look at this similation path by eye and can note that even with as much as 100 years of data we could easily interpret the series as being non-stationary when, in fact, the underlying model is stationary. This serves to highlight the fallacy of trying to prove or disprove stationarity.

The shorter-term volatility in $X_{1}(t)$ is mainly reflected in $r(t)$. This volatility also affects $\rho(t)$ but to a lesser extent and we can consequently observe that $\rho(t)$ is less volatile.

In Figure 4.8 we give a scatter plot of $r(t)$ versus $\rho(t)$ over the 400 years of the simulation, corresponding to Figure 1.2. Most of the points lie to the left of the diagonal $r(t)=\rho(t)$, indicating, as we remarked earlier that the yield curve is generally upward sloping (but possibly with a hump). We can note that the regression slope would be less than 1. Further empirical analysis of the model has indicated that this slope is very much dependent on the value of $\rho_{12}$, the correlation between $d X_{1}(t)$ and $d X_{2}(t)$. The more negative is $\rho_{12}$, the steeper is the regression slope.

\section{Extensions to a wider family: the Integrated Affine (IA) class}

We have worked here with a pricing formula which can be expressed in the form

$$
\begin{aligned}
P(t, T) & =\frac{A(t, T)}{A(t, t)}=\frac{\int_{T}^{\infty} e^{B(t, u)+C(t, u)^{\prime} X(t)} d u}{\int_{t}^{\infty} e^{B(t, u)+C(t, u)^{\prime} X(t)} d u} \\
\text { where } e^{B(t, u)+C(t, u)^{\prime} X(t)} & =\phi e^{-\beta t} H(u-t, X(t)) \quad(\phi=1 \text { say }) . \\
\text { Thus } B(t, u) & =-\beta u-\frac{1}{2} \sum_{i, j} \frac{\rho_{i j} \sigma_{i} \sigma_{j}}{\alpha_{i}+\alpha_{j}} e^{-\left(\alpha_{i}+\alpha_{j}\right)(u-t)} \\
\text { and } C_{i}(t, u) & =\sigma_{i} e^{-\alpha_{i}(u-t)} .
\end{aligned}
$$

This suggests an interesting extension to a much wider class of integrated affine (IA) models. For example, in the one-factor case we can propose a model for $P(t, T)$ as in equation (5.1) but where $d X(t)=\alpha(\mu-X(t)) d t+\sigma \sqrt{X(t)} d \hat{Z}(t)$. It is then necessary for us to establish the relevant functional forms for $B(t, u)$ and $C(t, u)$ to ensure that $\exp [B(t, u)+C(t, u) X(t)]$ is a $\hat{P}$-martingale (equivalent to $\phi(u) M(t, u)$ in Section 2).

Equally this can be extended to multifactor models. It is well known that the standard, multifactor affine term-structure models $P(t, T)=\exp \left[\tilde{B}(t, T)+\tilde{C}(t, T)^{\prime} Y(t)\right]$ exist for a limited range of diffusion processes for $Y(t)$ (see, for example, Duffie \& Kan, 1996). In the context of the IA models we ask the question: 
Risk-free Rate, $r(t)$

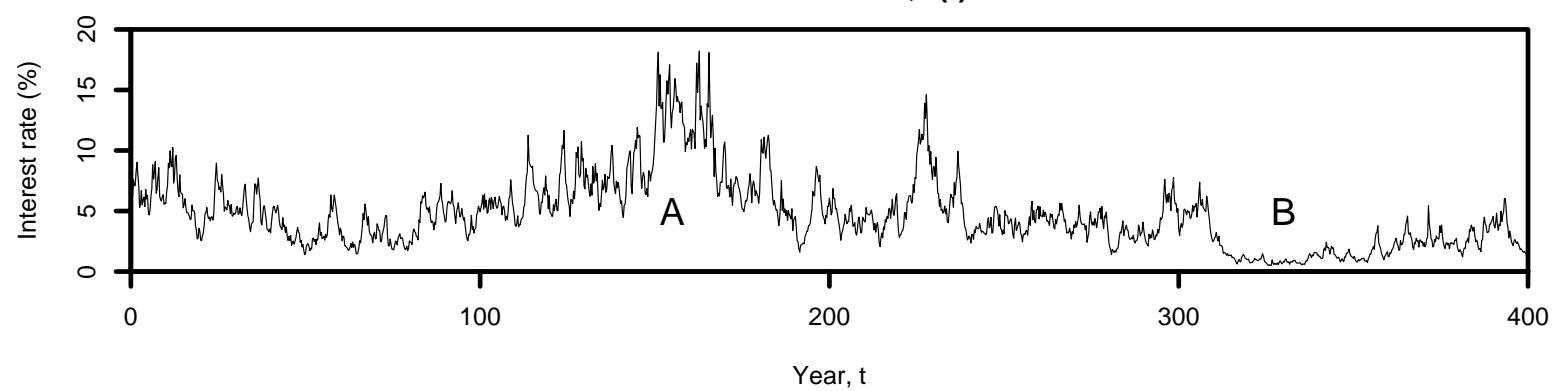

Consols rate

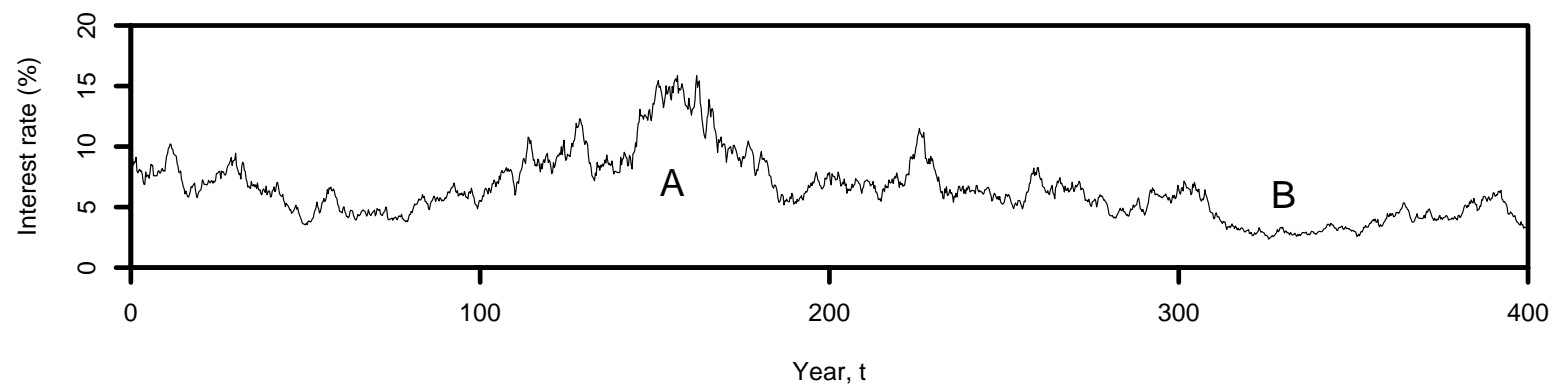

X_1(t)

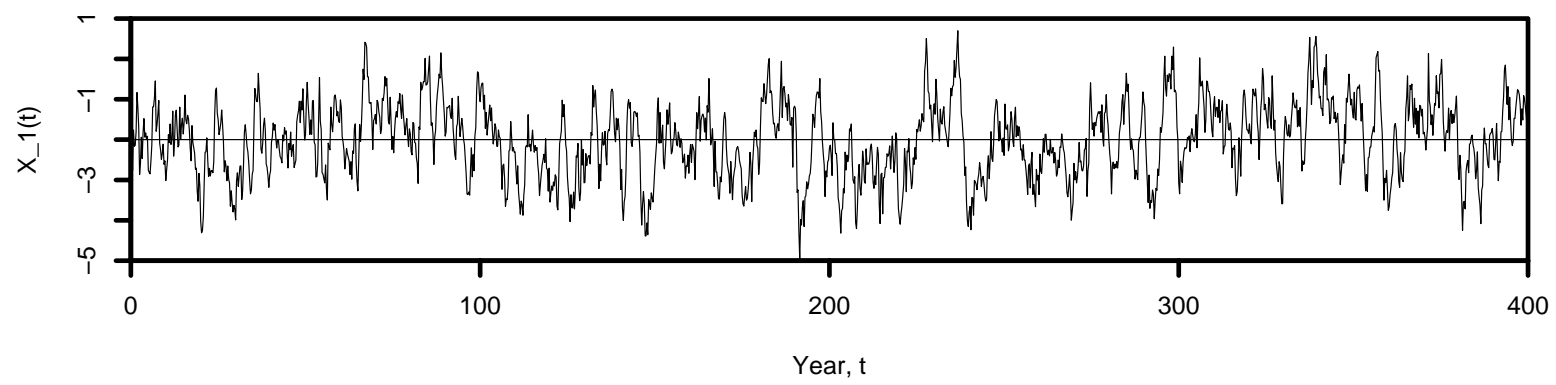

X_2(t)

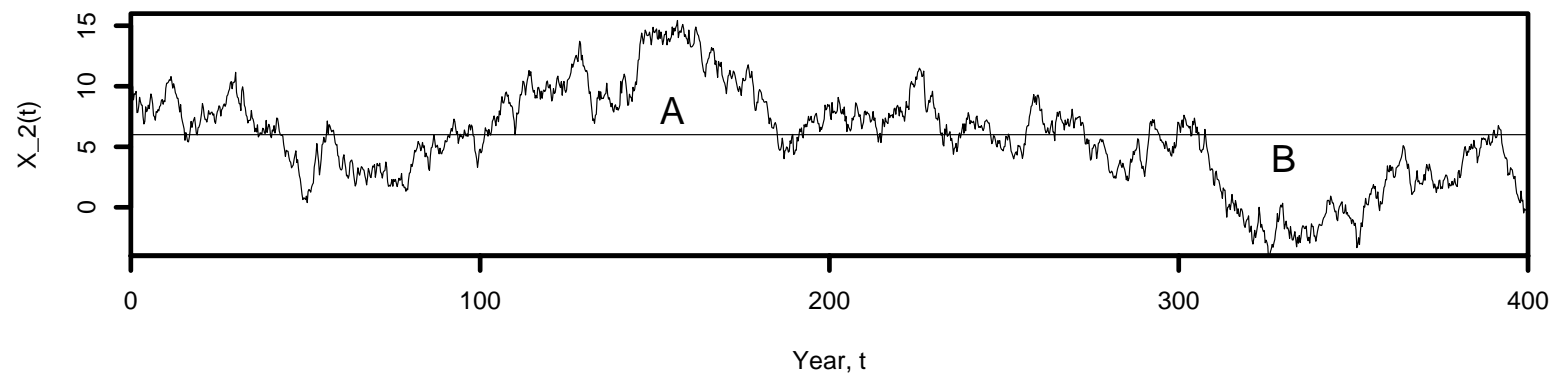

Figure 4.7: Simulation example for $\alpha=(0.6,0.06)^{\prime}, \sigma=(0.6,0.4)^{\prime}, \rho_{12}=-0.5$, $\beta=0.04$ and $\mu=(-2,6)^{\prime}$. In the plots for $X_{1}(t)$ and $X_{2}(t)$ the horizontal line indicates the mean-reversion level, $\mu_{i}$, for $X_{i}(t)$ under $P$. In the various plots $\mathrm{A}$ and B correspond to a peak and a trough in $X_{2}(t)$. 
5 EXTENSIONS TO A WIDER FAMILY:THE INTEGRATED AFFINE (IA) CLASS35

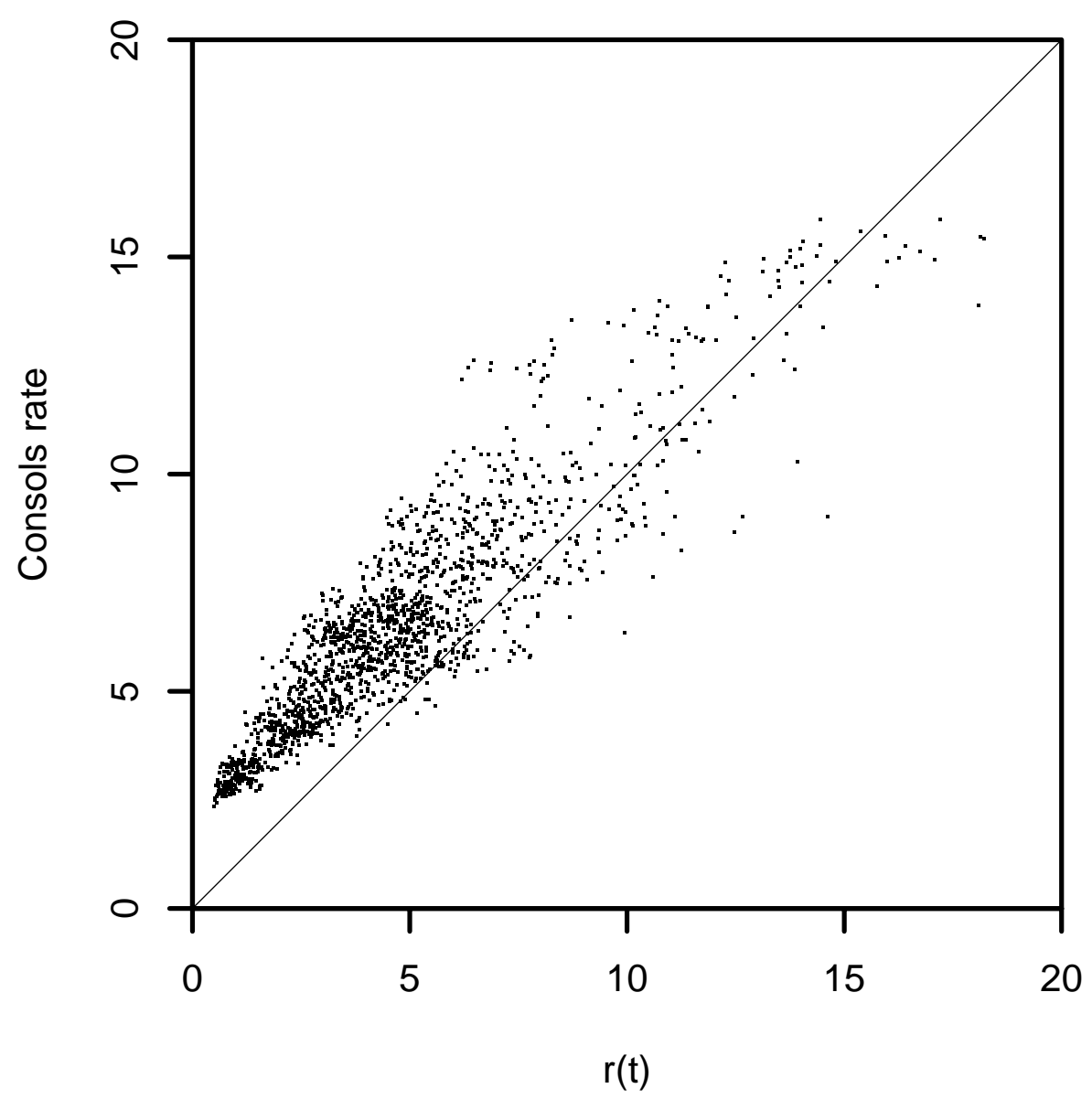

Figure 4.8: Simulation example for $\alpha=(0.6,0.06)^{\prime}, \sigma=(0.6,0.4)^{\prime}, \rho_{12}=-0.5$, $\beta=0.04$ and $\mu=(-2,6)^{\prime}$. Scatter plot of $r(t)$ versus the consols rate, $\rho(t)$. The diagonal line is $r(t)=\rho(t)$. 
for what $n$-dimensional diffusion processes, $X(t)$, do there exist deterministic functions $B(t, u)$ and $C(t, u)$ such that $\exp \left[B(t, u)+C(t, u)^{\prime} X(t)\right]$ is a martingale for all $u$ ?

We conjecture that $X(t)$ is limited to the same range of processes established by Duffie \& Kan (1996).

As with the affine term-structure class of models it is expected that the extension proposed here will give access to a wider range of volatility term structures.

\section{Conclusions}

We have proposed in this paper a new family of time-homogeneous multifactor models for the term structure of interest rates.

The primary motivation for this is to provide a powerful tool for long-term risk management and the pricing of long-term interest-rate derivatives. Theoretical aspects of the model were investigated first. More importantly though we have conducted a thorough numerical analysis of the 2-factor model in order to establish the suitability of the wider family for long-term risk management. These investigations have demonstrated that the 2-factor model, with a suitable choice of parameters, can satisfy all of the desirable characteristics set out in Section 1. In particular, the model can

- produce sustained periods of both high and low interest rates;

- produce wide ranges of values for both short and long-term interest rates, consistent with historical data;

- in extreme cases, give rise to yield curves similar to those experienced in Japan in 2002.

We have not attempted statistical fitting of the model to historical and market data. This is a considerable piece of research in its own right and is the subject of ongoing work.

Bond and derivative prices need to be calculated numerically. However, we have shown that bond prices require a simple one-dimensional numerical integration procedure. In the case of derivative pricing we will be able to exploit the normal distribution of the state variable, $X(t)$, to develop efficient pricing procedures. 


\section{Acknowledgements}

Many thanks go to a variety of workshop, conference and seminar audiences in Cambridge, Edinburgh, Lisbon, Manchester, Waterloo, Stockholm and Tokyo where this work has been presented. There have been many useful questions and comments which have helped with the presentation of the material. I am grateful to David Wilkie for providing the data in Figures 1.1 and 1.2. Numerical aspects of the model have been developed with the help of four excellent students on the MSc in Financial Mathematics: Gavin Falk, David Lonie, Nelius du Plessis and Paul Wilson. 


\section{References}

Black, F., and Karasinski, P. (1991) "Bond and option pricing when short rates are log-normal," Financial Analysts Journal July-Aug, 52-29.

Brace, A., Gatarek, D., and Musiela, M. (1997) "The market model of interest-rate dynamics," Mathematical Finance 7, 127-155.

Brown, R.H., and Schaefer, S. (2000) "Why long-term forward interest rates (almost) always slope downwards," Working paper: London Business School.

Cairns, A.J.G. (1998) "Descriptive bond-yield and forward-rate models for the British government securities' market," British Actuarial Journal 4, 265-321.

Cairns, A.J.G. (2003) Interest-Rate Models: An Introduction. Princeton University Press: Princeton. (Forthcoming.)

Chan, K.C., Karolyi, G.A., Longstaff, F.A., and Sanders, A.B. (1992) "An empirical comparison of alternative models of the short-term interest rate.," Journal of Finance 47, 1209-1227.

Constantinides, G.M. (1992) "A theory of the nominal term structure of interest rates," Review of Financial Studies 5, 531-552.

Cox, J., Ingersoll, J., and Ross, S. (1985) "A theory of the term-structure of interest rates," Econometrica 53, 385-408.

Duffie, D. (1996) Dynamic Asset Pricing Theory, 2nd Edition. Princeton University Press: Princeton.

Duffie, D., and Kan, R. (1996) "A yield-factor model of interest rates," Mathematical Finance 6, 379-406.

Dybvig, P., Ingersoll, J.E., and Ross, S.A. (1994) "Long forward and zero-coupon rates can never fall," School of Business, Washington University, St Louis.

Feldman, K.S., Bergman, B., Cairns, A.J.G., Chaplin, G.B., Gwilt, G.D., Lockyer, P.R., and Turley, F.B. (1998) "Report of the fixed interest working group," British Actuarial Journal 4, 213-263.

Flesaker, B., and Hughston, L.P. (1996) "Positive interest," Risk 9(1), 46-49.

Heath, D., Jarrow, R. and Morton, A. (1992) "Bond pricing and the term structure of interest rates: a new methodology for contingent claims valuation," Econometrica $60,77-105$.

Hunt, P.J., and Kennedy, J.E. (2000) Financial Derivatives in Theory and Practice. Wiley: Chichester.

James, J., and Webber, N. (2000) Interest Rate Modelling. Wiley: Chichester. 
Karatzas, I., and Shreve, S.E. (1998) Methods of Mathematical Finance. SpringerVerlag: New York.

Rebonato, R. (1998) Interest Rate Option Models, Second Edition. Wiley: Chichester.

Rogers, L.C.G. (1997) "The potential approach to the term-structure of interest rates and foreign exchange rates," Mathematical Finance 7, 157-164.

Rutkowski, M. (1997) "A note on the Flesaker \& Hughston model of the term structure of interest rates," Applied Mathematical Finance 4, 151-163.

Vasicek, O. (1977) "An equilibrium characterisation of the term structure," Journal of Financial Economics 5, 177-188.

Waters, H.R., Wilkie, A.D., and Yang, S. (2003) "Reserving and pricing and hedging for policies with guaranteed annuity options," To appear in the British Actuarial Journal.

Wilkie, A.D. (1995) "More on a stochastic asset model for actuarial use (with discussion)," British Actuarial Journal 1, 777-964.

Yakoubov, Y., Teeger, M., and Duval, D. (1999) "A stochastic investment model for asset and liability management," In Proceedings of the 30th International ASTIN Colloquium and 9th International AFIR Colloquium, August, 1999, J: 237-266. 